\title{
A Solute Gradient in the Tear Meniscus. I. A Hypothesis to Explain Marx's Line
}

\author{
Anthony J. Bron, FRCS, FMedSci, ${ }^{1}$ Norihiko Yokoi, MD, PhD, ${ }^{2}$ \\ Eamonn A. Gaffney, PhD, ${ }^{3}$ And John M. Tiffany, MA, PhD ${ }^{1}$
}

\begin{abstract}
Marx's line is a line of mucosal staining behind the mucocutaneous junction. It can be demonstrated throughout life in all normal lids by staining with lissamine green and related dyes. Of all the body orifices, only the mucosae of the eye and mouth are directly exposed to the atmosphere. In this paper, we suggest that for the eye, this exposure leads to the formation of Marx's line. The tear meniscus thins progressively toward its apex, where it is pinned at the mucocutaneous junction of the lid. It also thins toward the black line, which segregates the meniscus from the tear film after the blink. We predict that, because of the geometry of the tear meniscus, evaporation generates a solute gradient across the meniscus profile in the anteroposterior plane, which peaks at the meniscus apices at the end of the interblink. One outcome would be to amplify the level of tear molarity at these sites so that they reach hyperosmolar proportions. Preliminary mathematical modeling suggests that dilution of this effect by advection and diffusion of solute away from the meniscus apex at the mucocutaneous junction will be restricted by spatial constraints, the presence of tear and surface mucins at this site, and limited fluid flow. We conclude that evaporative water loss from the tear meniscus may result in a physiological zone of hyperosmolar and related stresses to the occlusal conjunctiva, directly behind the mucocutaneous junction. We hypothesize that this stimulates a high epithelial cell turnover at this site, incomplete epithelial maturation, and a
\end{abstract}

\footnotetext{
Accepted for publication February 2011.

From the ${ }^{1}$ Nuffield Laboratory of Ophthalmology, University of Oxford, Oxford, UK, ${ }^{2}$ Department of Ophthalmology, Kyoto Prefectural University of Medicine, Kyoto, Japan, ${ }^{3}$ The Mathematical Institute, University of Oxford, Oxford, UK

The authors have no proprietary or commercial interest in any product or concept discussed in this article.

Single-copy reprint requests to Anthony J. Bron, FRCS, FMedSci (address below).

Corresponding author: Anthony J. Bron, FRCS, FMedSci, Nuffield Laboratory of Ophthalmology, Walton Street, University of Oxford, Oxford OX26AW, UK. E-mail: Anthony.Bron@eye.ox.ac.uk.
}

O2011 Ethis Communications, Inc. The Ocular Surface ISSN: 1542-0124. Bron AJ, Yokoi N, Gaffney EA, Tiffany JM. A solute gradient in the tear meniscus. I. a hypothesis to explain Marx's line. 2011;9(2):70-91. failure to express key molecules such as MUC 16 and galectin-3, which, with the tight junctions between surface epithelial cells, are necessary to seal the ocular surface and prevent penetration of dyes and other molecules into the epithelium. This is proposed as the basis for Marx's line. In Part II of this paper (also published in this issue of The Ocular Surface), we address additional pathophysiological consequences of this mechanism, affecting lid margins.

KEY WORDS evaporation, fluorescein, galectin-3, lid margin, lissamine green, Marx's line, meibomian gland dysfunction, MGD, mucin, mucocutaneous junction, osmolarity, rose bengal, solute, tear meniscus

\section{INTRODUCTION}

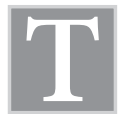
he palpebral aperture is a body orifice that performs an essential function, permitting light to enter the eye in the waking state. While achieving this, the surface of the eye is open to the atmosphere and is at risk of dehydration. Other such orifices, lined by mucous membrane and interfacing with skin at mucocutaneous junctions (MCJs), are the mouth, the nose, the lacrimal puncta, and the nipple, vagina, urethra, and anus. Often, at each location, a transitional epithelial layer is interposed between the mucosa proper and the skin, but only the vermilion of the mouth is constantly exposed to the atmosphere. The mucosae of the other orifices are protected by orificial closure.

The mucosae of the ocular surface, represented by the conjunctiva and the cornea, are exposed directly to the ambient air and are therefore at risk of desiccation through water loss. However, protective mechanisms are in place, such as the presence of the tear film and of tear flow and blinking, which reduce drying. The physiological means by which the eye is protected are well understood, but when they fail, dry eye results and tear hyperosmolarity is the central mechanism for tissue damage. ${ }^{1}$ In this article, we introduce a novel mechanism that could amplify desiccating stress to eye, focusing damage to the lid margin.

The tear fluid of the eye is located in the tear menisci and tear film, together comprising the preocular compartment, and in the fornical compartment, behind the lids and in the fornix (Figure 1). When the eyes are closed, the preocular 


\section{OUTLINE}

I. Introduction

II. The anatomy of the lid margin
A. The cutaneous surface of the outer lid
B. The ciliary zone
C. The cutaneous occlusal zone
D. The mucocutaneous junction
E. The marginal zone
F. Epithelial biomarkers in skin, mucocutaneous junction and mucosa

III. The permeability of the conjunctival and corneal epithelia

IV. Location of stem cells for the marginal conjunctiva

V. Variation of osmolarity in the tear compartments

VI. The tear menisci

VII. Staining of the ocular surface with topical dyes
A. Historical aspects
B. Rose bengal and lissamine green
C. Fluorescein sodium

VIII. Marx's line
A. Background
B. Etiology of Marx's line

1. An intrinsic property of the occlusal cells of the mucosa

2. Mechanical trauma

3. Desiccation

4. A solute gradient to explain Marx's line: A new hypothesis
a. Pinning of the meniscus apex
b. The geometry of the meniscus
c. Physicochemical properties of the meniscus
d. Physicochemical interactions between solutes present in the tears
e. Physicochemical interactions between solutes and the glycocalyx

C. Events predicted by the gradient hypothesis during the interblink

D. An explanation for Marx's line, based on the gradient hypothesis

E. Potential modifiers of Marx's line

IX. Summary and conclusions

compartment disappears. In earlier papers, we have suggested that when the eyes are open, tear osmolarity is not uniformly distributed between the tear compartments, but differs between the tear menisci, film, and fornix, with the level being lower in the meniscus than in the tear film. ${ }^{2}$ This has been supported by mathematical modeling. The differential is small in the normal subject, but is predicted to increase in dry eye disease. ${ }^{3}$

In the current paper, we carry this concept further, suggesting that osmolarity is also unevenly distributed within the tear menisci and is highest at the peripheral edge or apex of each meniscus, where it makes contact with the MCJ. While the arguments put forward apply in some degree to other menisci formed at the ocular surface (eg, at the edge of a hard contact lens), only the apex at the mucocutaneous junction is spatially pinned and could continuously subject the underlying epithelial cells to osmolar or other solute stress.

We hypothesize that this phenomenon is the basis for the physiological staining pattern called Marx's line. The further consequences of this hypothesis, providing a mechanism for the progression of lid margin disease in dry eye and other inflammatory disorders, is detailed elsewhere in this issue. ${ }^{4}$ The arguments for this gradient hypothesis are presented below.

To understand how this mechanism might influence physiological and pathological events at the lid margin, it is necessary to describe selected features of lid anatomy and physiology and certain methods of clinical assessment of the external eye.

\section{THE ANATOMY OF THE LID MARGIN}

In this report, we consider the lid margin to include not only its appositional or occlusal surface, which makes contact with that of the opposing lid during eye closure, but also the distal $2 \mathrm{~mm}$, which permits the inclusion of the lash line anteriorly and the subtarsal fold on the posterior surface of the tarsal plate (Figure 2). The anterior lid margin is skin-covered and gently curved, convex outwards, while its posterior margin, lined by a specialized conjunctiva, is curved more acutely, at about a right angle to the occlusal surface. The lid margin is about $2 \mathrm{~mm}$ wide at the center of the lower lid, measured from the posterior lash line to the posterior lid margin. ${ }^{5}$

The salient aspects of human lid margin anatomy have been described by Marx, ${ }^{6,7}$ Wolff, ${ }^{8}$ Munger and Halata, ${ }^{9}$ and Knop et al. ${ }^{10}$ An English translation of the original Marx article has recently been published. ${ }^{11}$ The sensory innervation of both the human and the primate (rhesus monkey) eyelid has also been described in some detail., ${ }^{9} 12$ We have modified the nomenclature used by these authors to recognize that the marginal mucosa of the posterior lid margin, best regarded as a transitional epithelium, presents separate features 1) on the occlusal surface of the lid and 2) on the posterior surface of the tarsal plate. We have adopted this approach in order to emphasize that these distinct zones encounter different physiological stresses and most likely have different functional roles.

In sagittal section, the lid margins exhibit five distinct regions, which are, from front to back, 1) the cutaneous surface of the outer lid, 2) the ciliary zone, 3) the occlusal skin, representing the cutaneous part of the appositional surface of the lid margin, 4) the mucocutaneous junction, and 5) the marginal zone of the conjunctiva. Proximally, the epithelium of this latter zone is in continuity with the tarsal conjunctiva proper, which is continuous, in turn, with the orbital, fornical, and bulbar conjunctiva, in that order. ${ }^{13}$ The details are described below (Figure 2).

\section{A. The Cutaneous Surface of the Outer Lid}

The skin of the outer lid has a thin, squamous, keratinized epidermis, which bears fine, scattered, vellus hairs like 
Figure 1. Tear compartments of the eye. When the eyes are open, the tears are distributed in three compartments, the tear menisci and tear film, which together make up the preocular tears, and the fornical compartment, which comprises tear fluid in the fornices and behind the lids. (Reprinted from Gaffney EA, Tiffany JM, Yokoi N, Bron AJ, et $\mathrm{al}^{3}$ with permission of Prog Retin Eye Res.)

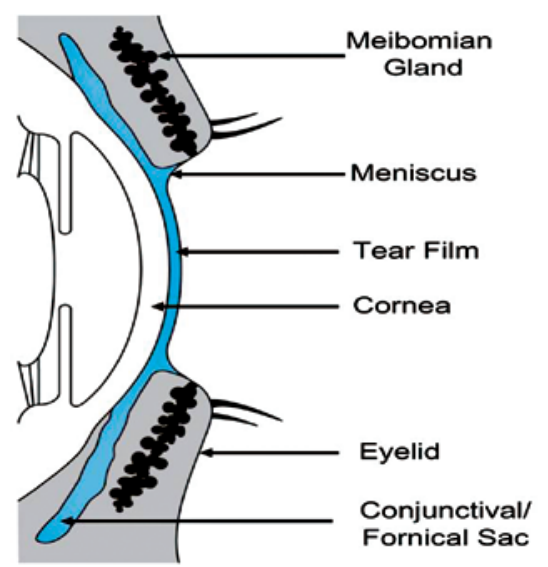

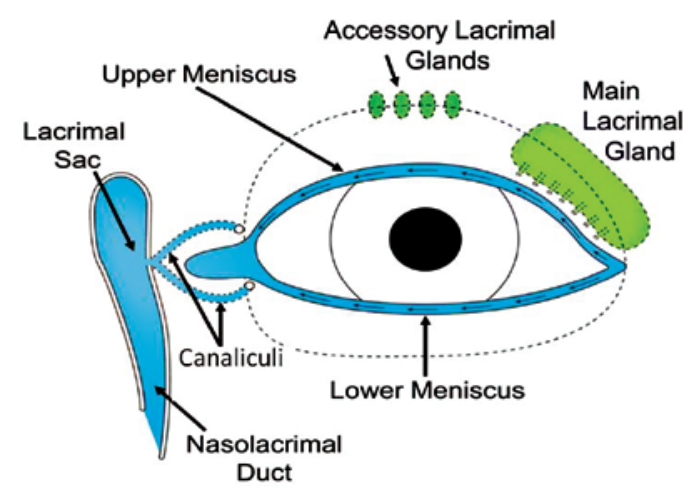

those of the facial skin. ${ }^{9}$ This skin exhibits fine undulations corresponding to the skin lines, and low rete pegs and dermal papillae. Unlike the other zones of the lid margin, the dermis is loosely attached to underlying connective tissue. Therefore, this skin can be readily rolled between the fingers and is billowed out in the presence of edema fluid. In contrast, the appositional zones of the lid margin and the mucosa of the tarsal plate are tightly attached to the underlying connective tissues of the lid, presumably equipping them to deal with physical stresses, such as the impact of the blink and the frictional forces associated with the eye movements and blinking.

\section{B. The Ciliary Zone}

The ciliary, or eyelash-bearing, region of the lid margin has a thick epidermis with tall rete pegs and broader dermal papillae. Each lash follicle receives sebaceous secretions from the adjoining glands of Zeiss, each of which possesses

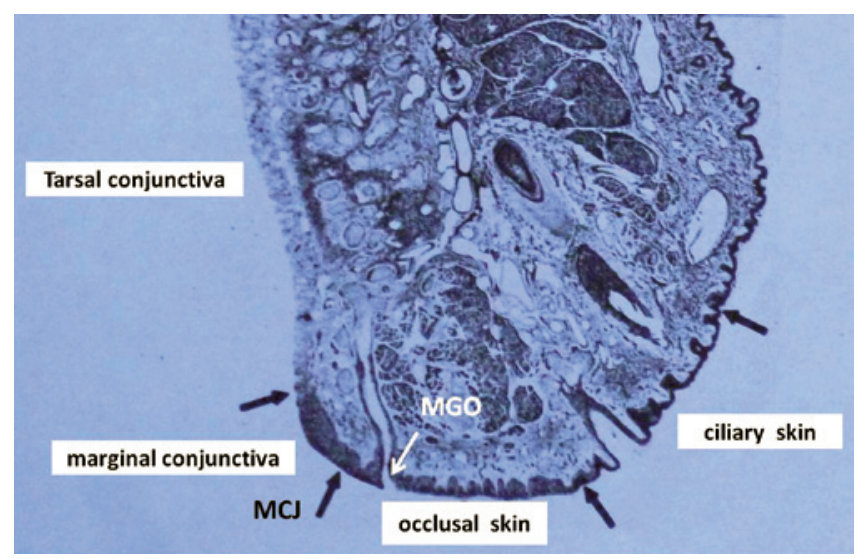

Figure 2. Cross-sectional view of the human eyelid showing the organization of epithelium at the lid margin. To the right is the keratinized, stratified squamous epidermis of the ciliary region, penetrated by the lash follicles. Centrally is the epidermis of the occlusal skin, which extends posteriorly to the mucocutaneous junction (MCJ) and embraces the orifices of the meibomian glands (MGO). Directly behind the MCJ is the nonkeratinized, stratified squamous marginal epithelium of the conjunctiva, which possesses a narrow, occlusal zone anteriorly and a global zone on the posterior surface of the tarsal plate. The global zone gives way to the tarsal conjunctiva proper at the sub-tarsal fold. (Reprinted from Munger $\mathrm{BL}$, Halata $\mathrm{Z}^{9}$ with permission of $\mathrm{Am} J$ Anat).
1-3 acini, compared to $10-20$ in the ordinary sebaceous glands of the skin. Additionally, the eccrine glands of Moll are present, in relation to the lash bulbs. ${ }^{8}$

\section{The Cutaneous Occlusal Zone}

The cutaneous occlusal zone extends from the ciliary zone to the MCJ, where the epidermis gives way to the epithelium of the marginal conjunctiva. The epithelium of the conjunctiva is situated on a higher level relative to the epithelium of the epidermis of the eyelid. ${ }^{6}$ This is visible clinically by slit lamp microscopy ${ }^{11}$ or, as we have observed, by specular microscopy of the tear meniscus. Marx has attributed this elevation to the abrupt ending of the stratum granulosum and corneum, which reappear in a different shape, and thicker, at the start of the conjunctiva. ${ }^{6,14}$

The epidermis comprises a thick, keratinized, stratified squamous epithelium, with blunt rete pegs and tall but narrow dermal papillae. Roughly midway between the posterior lash line and the MCJ is the grey line, which represents the dermal locus of insertion of Riolan's part of the orbicularis muscle. ${ }^{6,15}$ It divides the lid into an anterior lamella (skin and muscle) and a posterior lamella (tarsus and conjunctiva) between which there is a relatively avascular plane. ${ }^{17}$

The orifices of the meibomian glands, some 20-30 in the upper lid ${ }^{13,18}$ and about 24 in the lower lid, emerge through lid skin in a continuous row, parallel to the MCJ and slightly anterior to it. In some individuals, orifices may be staggered anteroposteriorly, ${ }^{5}$ and in older subjects the course may become irregular as a result of pathological changes. The orifices lie roughly $810 \mu \mathrm{m}( \pm 160)$ apart, directly anterior to the MCJ and separated from it by a distance of about $180 \mu \mathrm{m}$ in normal lids. ${ }^{19,20}$ Their location ensures that meibomian oil is delivered to the hydrophobic skin of the lid margin, where it forms a shallow reservoir. From here it is delivered onto the tear menisci and tear film in the upstroke of each blink to form the tear film lipid layer. ${ }^{21}$

On biomicroscopy, each meibomian gland orifice is surrounded by a series of rings, probably representing, from within outward, the partially keratinized lining of the terminal duct ${ }^{22,23}$ and externally, the outer edge of the circular floret of the meibomian acinus with its connective tissue sheath, viewed through the epidermis. ${ }^{24,25}$ Fibers 
from the muscle of Riolan may also make a contribution. The MCJ is located immediately behind the perimeter of this region (Figure 3 ).

The keratinized epidermis of the lid margin skin is, like skin elsewhere in the body, hydrophobic and therefore not wettable by the tears. It is normally coated by lipid secretions, anteriorly by sebum from the sebaceous glands of the skin and the glands of Zeiss, and posteriorly by meibomian oil. The degree to which these two lipids mix on the lid margin is not known, but it is thought that the meibomian oil prevents sebum from reaching and contaminating the tear film and destabilizing the tear film lipid layer. ${ }^{24}$

\section{The Mucocutaneous Junction}

Biomicroscopically, the MCJ is seen as a sharp line of junction between the occlusal skin and the occlusal conjunctiva (see below), which in a young person takes a fairly smooth, curvilinear course along the length of the lid, parallel to the posterior lid margin. Marx observed that the junction is perceptibly raised above the plane of the occlusal epidermis, and we have confirmed this by specular microscopy. The sharp boundary is emphasized by the appearance of the meniscus apex when it is viewed by specular microscopy or with fluorescein dye, and this inserts directly behind the MCJ. This apex demarcates the hydrophobic skin

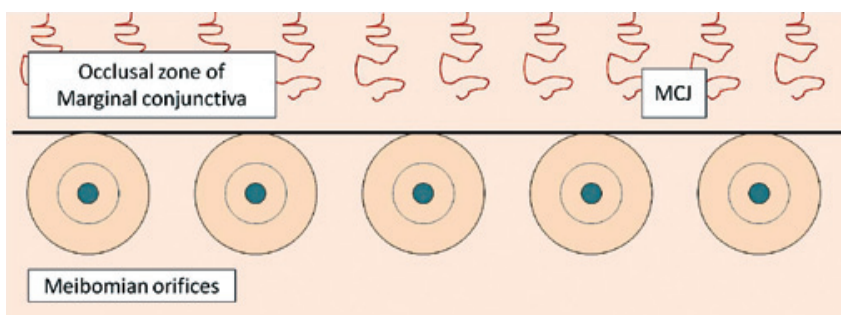

Occlusal skin

A

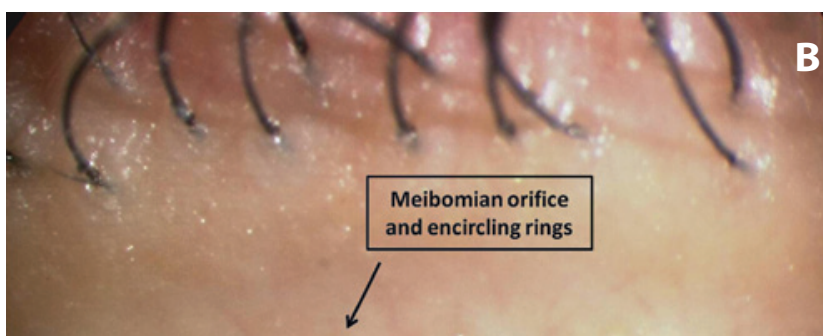

Posterior lid margin

Occlusal conjunctiva

Figure 3. A. Diagram of the occlusal surface of the lid margin. In the young normal lid, the meibomian gland orifices and surrounding structures are directly anterior to the mucocutaneous junction. B. Slit-lamp view of the normal lid margin in a young adult, showing the posterior lid margin, occlusal part of the marginal conjunctiva, and the disposition of the meibomian orifices. from the hydrophilic conjunctiva, so that the junction is characterized by the abrupt change in water wettability of the two zones. Nasally and temporally, the mucocutaneous junctions of the upper and lower lid join with those of their fellow lids. Nasally, the MCJs pass anterior to the puncta, which are surrounded by conjunctiva and therefore bathed by the aqueous of the tear lake.

It is less certain that the MCJ exists as a clearly identifiable sharp line of junction at the histological level in the absence of specific biomarkers. Riau et al, in the mouse, represent it as a zone a few cells wide. ${ }^{10,27}$ Knop et al noted that the epithelium that follows the abrupt cessation of the keratinized layers of the epidermis are still stratified squamous but are nucleated and contain a dense cytoplasm, entitling them to be regarded as parakeratinized. ${ }^{28}$ They consider that these parakeratinized cells correspond to the cells staining as Marx's line. ${ }^{28}$

After the age of about 50 years, the MCJ becomes increasingly irregular, $5,19,20,29,30$ and in the presence of various forms of blepharitis, it may migrate either forward or backward. ${ }^{29,31,32}$ Forward migration of the MCJ causes it to lie anterior to the meibomian orifices in a process sometimes referred to as "conjunctivalization" of the lid margin. ${ }^{33,34}$ Posterior migration of the MCJ is a prominent feature of cicatricial conjunctivitis, but it also occurs to a lesser degree as a primary disorder or secondary to skin disease. ${ }^{31,32} \mathrm{In}$ the early stages, this scarring causes narrowing of the occlusal zone of the marginal mucosa and ultimately drags the MCJ onto the posterior tarsal plate, where it is ultimately absorbed. Posterior migration is usually accompanied by retroplacement of the meibomian orifices themselves, which come to lie behind the mucocutaneous junction. This renders them functionally ineffective, even if they are not obstructed. This condition is termed cicatricial meibomian gland dysfunction and contrasts with non-cicatricial meibomian gland dysfunction, where, initially, the meibomian orifices retain their normal location anterior to the MCJ. ${ }^{31,32,34}$ As noted above, forward migration of the MCJ may also place the affected orifices deep to the tear meniscus, but with the orifices remaining on the occlusal surface of the lid margin.

\section{E. The Marginal Zone}

The marginal conjunctiva is a transitional zone between the skin and conjunctiva proper. It extends posteriorly from the MCJ for about $2 \mathrm{~mm}$, beyond the posterior edge of the lid margin and onto the tarsal plate, where it ends at the subtarsal fold (Figure 2). ${ }^{8}$ Here, the stratified squamous, nonkeratinized epithelium gives way abruptly to the columnar/cuboidal architecture of the epithelium, covering the remainder of the tarsal plate. ${ }^{8,13}$ The marginal conjunctiva, like other conjunctival regions and mucosal surfaces in general, is hydrophilic and is normally bathed by aqueous tears, which maintain it in its moist and healthy state. The term conjunctiva proper will be used to refer to all other conjunctival regions, ie, excluding the marginal conjunctiva.

For descriptive purposes, particularly in relation to the 
formation of Marx's line, it is useful to divide the marginal conjunctiva into 1) a narrow occlusal zone, directly behind the MCJ and located on the appositional or free border of the lid, and 2) a global zone, on the posterior surface tarsal plate, which is in direct contact with the globe. The two zones are in continuity at the edge of posterior lid margin, also referred to as the crest (Figure 2). ${ }^{28}$ It is the occlusal surfaces of the upper and lower lid that are apposed during lid closure, whether in sleep or, more briefly, at the end of the down-stroke of the blink.

Parsons observed that the marginal conjunctiva was in close contact with the globe and was aware that this squamous epithelium continued onto the posterior tarsal plate, up to the subtarsal fold. ${ }^{18}$ Ehlers was similarly aware of the interface between the marginal conjunctiva and the goblet cell-containing tarsal conjunctiva on the posterior surface of the lid. ${ }^{35} \mathrm{He}$ concluded that the squamous epithelium of the marginal conjunctiva rubbed against the globe during the blink. Since the lower lid moves only slightly during the blink, this observation relates mainly to the upper lid. These authors were evidently referring to the global part of the marginal conjunctiva and did not specifically attempt to distinguish the occlusal and global parts of this region. This has led to some confusion in the literature, leading to the suggestion that Marx's line could arise through frictional contact between the lid margin and the globe (ie, the cornea or bulbar conjunctiva). ${ }^{20}$ However, since Marx's line is located on the occlusal surface of the lid, this could not occur.

Kessing provided further information about the apposition of the lids to the globe in a tomographic study in which the conjunctival sac was viewed in section after instillation of barium sulfate. ${ }^{36} \mathrm{He}$ concluded that while the entire posterior surface of the lower lid was in contact with the globe, this was only achieved by the posterior, marginal zone of the upper lid. It cannot be determined from these studies whether the area of contact included any of the tarsal conjunctiva proper, but it can be assumed to include, at least, the global part of the marginal conjunctiva. A different account of this architecture is given by Knop et al, who describe the mucosa stretching from the crest of the posterior lid margin to the subtarsal fold, as a goblet cell-containing conjunctiva forming the cushion of epithelial cells that has been termed the lid wiper (see below). ${ }^{10}$

The muscular force that maintains apposition between the lid margins and globe is thought to be provided by Riolan's muscle, a distinct muscle grouping, separate from the pretarsal orbicularis oculi muscle, which may have an additional role in regulating the delivery of meibomian oil. In the upper lid, it comes into play particularly during the blink. It is located at and lies parallel to the lid margin and consists of two separate bundles. ${ }^{37}$ The pars ciliaris lies anterior to the tarsal plate, and the pars subtarsalis, a smaller muscle, lies posterior to the orifices of the meibomian glands. They are joined together by interlacing fibers, the pars fascicularis, which traverse the tarsal plate and pass between the meibomian glands. The three bundles are presumed to act as a functional unit.
There is no evidence to suggest that the occlusal strip of marginal conjunctiva is apposed to the cornea or globe during the blink, other than at its most posterior edge (the crest), where it blends with the global zone. It is this global zone of the upper lid that has been named the lid wiper region of the lid. ${ }^{38}$ This region may, in part, take up stain with fluorescein and rose bengal in some normal eyes and, more particularly, in soft contact lens wearers and in dry eye patients, giving rise to the condition of lid wiper epitheliopathy (LWE). ${ }^{38,39}$ The disorder is thought to be due to excessive stresses, tangential and normal, to the lid wiper zone, as it passes across the underlying globe or a contact lens. LWE is also associated with partial blinks. ${ }^{40}$ Shiraishi et al described a parallel staining pattern affecting the marginal conjunctiva of the lower lid in a large population of normal Japanese subjects. ${ }^{41}$ This LWE-like appearance was present in $95.8 \%$ of individuals with upper lid LWE, but was actually 2.5 times more common than LWE itself in this population. If LWE is, in part, attributed to stresses generated by the movement between the lid margin and the globe during blinking, it might seem curious to encounter it in relation to the lower lid margin, which moves only slightly during the blink. However, stresses would also be generated at the lid wiper region and globe during eye movements, such as the saccade, and this should be considered as another possible etiological factor.

The epithelium of the marginal conjunctiva, unlike that of most of the conjunctiva proper, contains no goblet cells. ${ }^{8,18,35}$ In this respect, it resembles the two triangular islands of conjunctiva, located on either side of the nasal and temporal limbus in the horizontal meridian, which also lack goblet cells (Figure 4). ${ }^{36}$ Here, there is a discrepancy between standard descriptions. Wolff ${ }^{8}$ and Ehlers ${ }^{35}$ report the absence of goblet cells, while Munger and Halata refer

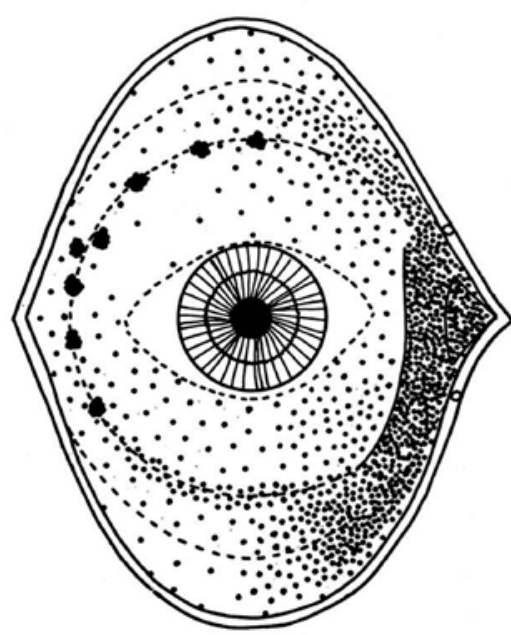

Figure 4. The goblet cell distribution in the human conjunctiva (round dots). Note that the density of glands is greatest inferonasally and on the caruncle, and least temporally. A goblet cell-free zone is present on either side of the limbus in the horizontal meridian. The more globular structures in the superotemporal fornicies are the accessory lacrimal glands. (Reprinted from Kessing SV ${ }^{36}$ with permission of Acta Ophthalmol [Copenh].) 


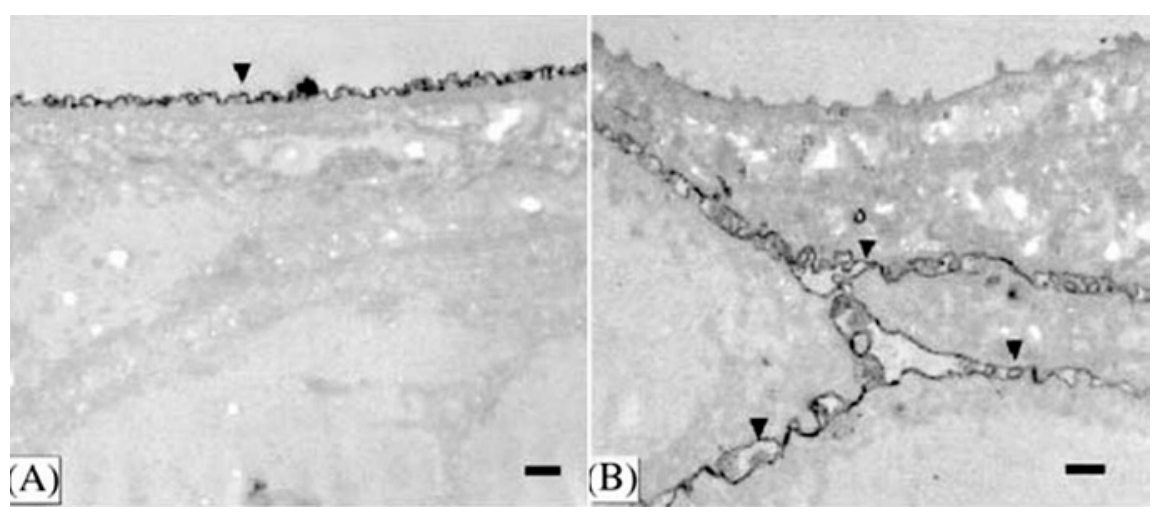

Figure 5. The barrier function of human corneal epithelial cells grown on amniotic membrane with and without air-lifting. In confluent, air-lifted epithelium (A), the tightjunctional barrier is intact and excludes the passage of horse radish peroxidase (HRP) tracer into the epithelium. In non-air-lifted epithelium (B), the barrier is absent and HRP passes readily between the apical cells and deeper cell layers of the epithelium. (Reprinted from Ban Y, Cooper LJ, Fullwood NJ, et $\mathrm{al}^{45}$ with permission of Exp Eye Res.) to a "glandular epithelium" but do not specify further. ${ }^{9}$ Proximal to the marginal conjunctiva is the conjunctiva of the tarsal plate, whose epithelium consists of one or two layers of columnar or cuboidal cells, replete with goblet cells. In the study by Shiraishi et al, impression cytology of the LWE and LWE-like regions showed a similar "goblet cell loss" and squamous metaplasia, ${ }^{41}$ but, as noted, the marginal conjunctiva is normally deficient in goblet cells.

\section{F. Epithelial Biomarkers in Skin, Mucocutaneous Junction and Mucosa}

Riau et al, recognizing parallels between mucocutaneous junctional regions in many parts of the body, examined the expression of biomarkers in the skin and mucosa and the MCJ of the lip and lid margin of the mouse. ${ }^{27}$ The marginal zone of the lid margin may be regarded as equivalent to the vermillion of the lips. The distribution of cytokeratins and other biomarkers was similar across the junctional regions studied, but not identical. They may be summarized as follows. In the two regions, CK1, CK 10 and filaggrin were absent in the mucosal epithelia, while the CK 4, CK6 and CK13 were absent from the epidermis. CK5 and CK 14 were present in the epidermis, MCJ, and mucosa. In the skin of each region, CK 19 was expressed basally, while involucrinpositive cells were found superficially in the skin, MCJ, and mucosa. Connexin 43 , which was present in all zones in the lips, was absent from the palpebral conjunctiva.

This study indicates some overlap in the expression of biomarkers for skin and conjunctiva in this transitional zone and perhaps gives some insight into the different functional requirements of the skin and mucosa. If these findings are confirmed in the human lid, they offer opportunities to better characterize the location of the MCJ.

\section{THE PERMEABILITY OF THE CONJUNCTIVAL AND CORNEAL EPITHELIA}

Marx's line is a region of paradoxical staining of the occlusal conjunctiva, and it is therefore relevant to explore the factors that influence the uptake of dyes into the epithelia of the ocular surface.

The corneal epithelium and, to a lesser extent, the bulbar conjunctival epithelium, ${ }^{42,43}$ form an obstruction to the passage of fluids, electrolytes, small water soluble solutes and macromolecules from the tears into the submucosa, by way of the natural barrier formed by the apical cell membranes of the surface epithelial cells and the tight junctions that exist between contiguous surface cells. ${ }^{4-46}$ The glycocalyx plays an additional role (Figure 5). It has been estimated in the rabbit that the corneal epithelium is up to 30-50 times less permeable than the conjunctiva. ${ }^{42,47}$ For the cornea, these confer the properties of an almost perfect semipermeable membrane. Both epithelia are permeable to lipid soluble molecules, such as chloramphenicol, some sulphonamides, the fluoroquinolones, and azithromycin. ${ }^{48}$ The permeability properties of the tarsal and marginal conjunctiva have not been studied and cannot be presumed to be identical to those of the bulbar conjunctiva. Deeper cells of the corneal epithelium, connected by desmosomes, are separated by tortuous intercellular spaces, which provide a paracellular route to the underlying stroma. The intercellular spaces are wider in the conjunctival epithelium. ${ }^{13,42}$

Tight junctional complexes $(\mathrm{Tj} s)$ of the corneal epithelium consist of the transmembrane proteins, occludin, claudin and the junctional adhesion molecules, and the peripheral membrane proteins, ZO- 1,-2 and - 3 and MUPP$1{ }^{49,50}$ Their distribution in the human corneal epithelium is illustrated in Figure 6. The occludins and claudins contain four transmembrane domains, with both their $\mathrm{N}$ - and Ctermini directed toward the cytoplasm. Occludin is combined with tissue-specific members of the claudin family to form paired strands, one from each adjacent cell, that cross between these cells and close the intercellular space. ${ }^{51}$ Occludin functions as a regulatory protein controlled by phosphorylation and has no significant structural role. ${ }^{52} \mathrm{It}$ may have permeability-related function $s^{53}$ and influence cell division. ${ }^{54}$ The ZO-proteins are members of the membraneassociated guanylate kinase proteins (MAGUK) and are located at membrane contact points of the Tjs. Here they form a complex that bridges between the $\mathrm{Tj}$ occludins and claudins and the actin cytoskeleton of the cell, which may be influenced by extracellular stimuli.

The claudins constitute a family of $(23 \mathrm{kDa})$ proteins, which are the only junctional proteins to show tissue specificity. Yoshida et al have summarized the role of the claudins as structural components of Tjs in the corneal and the bulbar conjunctival epithelia. ${ }^{46}$ Despite the differences 

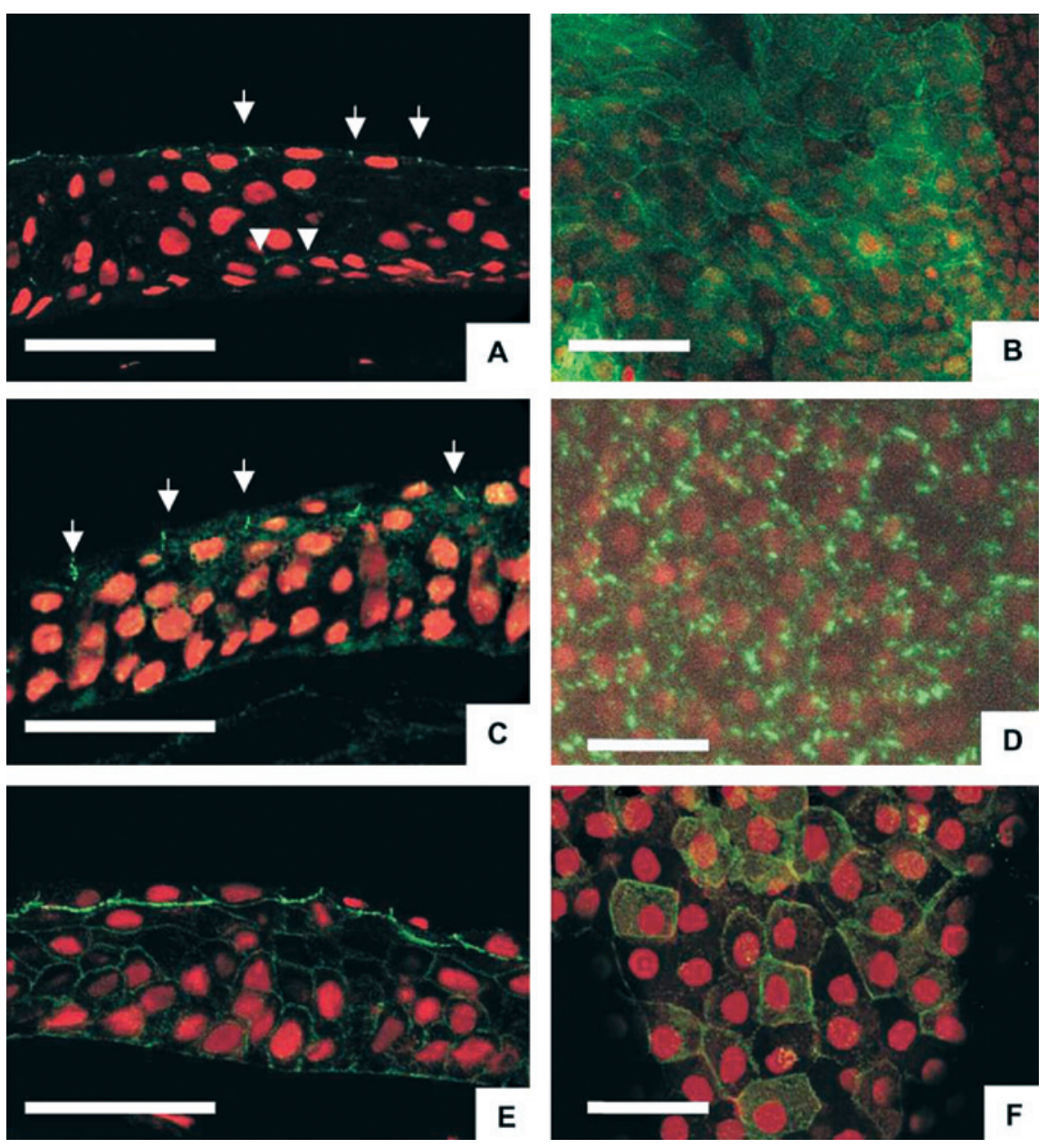

Figure 6. The distribution of tight-junctional components in human corneal epithelium shown by immunohistochemical staining. ZO-1 (A and B), occludin (C and D) and claudin-1 (E and $\mathbf{F})$, in transverse sections (A, C, E) or en face (B, D, F and H). Most apical cells of the epithelium exhibited ZO-1 immunoreactivity at their lateral margins (A; arrow). In en face images, ZO-1 forms a continuous ring around the large superficial epithelial cells (B). The staining pattern for occludin was concentrated at cell-cell borders, in the superficial layer of cells only (C). In en face images, staining was not continuous, but was dotted along the cell borders (D). Both basal and basolateral membranes of superficial cells were stained by claudin-1 antibody, but the apical membrane was not (E). In en face sections, claudin-1 antibodies showed as bands that corresponded to the basolateral membranes (F). No staining was observed in negative controls (not shown). Scale bar $1 / 4100 \mathrm{~mm}$. (Reprinted from Ban Y, Dota A, Cooper LJ, et al ${ }^{50}$ with permission of Exp Eye Res.) in permeability between these two epithelia, they found that each epithelium expressed the same claudin subtypes, namely claudins 1,4 and 7, throughout their thickness, although the expression of type 7 was superficial in the conjunctival epithelium. They postulated that the ratios of claudins and their phosphorylation state determined the tightness of Tjs in these apical surface cells. They suggested, too, that the presence of the same claudin subtypes in the subapical cell layers of these epithelia reflected a need to rapidly assemble the components of tight junctions in preparation for cell desquamation.

Claudin- 1 is ubiquitous and is found in the skin, where, together with claudin-4, it is located within encircling Tjs between contiguous keratinocytes of the epidermal granular layer. This contributes to the resistance of the skin to water loss. In keeping with this, claudin-4-deficient mice die after the first postnatal day from excessive water loss. ${ }^{51}$

The apical cell membranes of the surface epithelial cells and their associated glycocalyx offer a barrier to the transcellular diffusion of molecules from the tears. The plasmalemma is relatively impermeable to water-soluble molecules, and the associated glycocalyx forms an additional barrier, related at least to the expression of transmembrane mucins and to other molecules. This is discussed in a subsequent section.

\section{LOCATION OF STEM CELLS FOR THE MARGINAL CONJUNCTIVA}

It is important to the hypotheses advanced in this review to understand the source of the stem cells that maintain the conjunctival cells of the marginal conjunctiva. This is a topic over which there is still some controversy.

Stem cells may be defined as progenitor cells with a high capacity for cell division and the ability to generate a terminally differentiated progeny. ${ }^{55,56}$ This proliferative capacity is maintained for the lifetime of the individual and gives rise to a group of rapidly dividing, transient amplifying cells, which have a lower and limited, long-term proliferative capacity. They represent the largest group of dividing cells. Wei et al, using tritiated thymidine in the rabbit, demonstrated label-retaining cells at the limbus, fornix and lid margin, and concluded that the epithelial cells of the cornea and conjunctiva arose from two separate lineages. ${ }^{57,58}$ Label retained at the limbus was interpreted to identify the stem cells of the corneal epithelium, while, since label was abundant in the fornix, it was concluded that the fornix was a major site of conjunctival stem cells.

According to Pellegrini et al, based on a clonal analysis of conjunctival cells sampled from the limbus, bulbar conjunctiva and lid margin, stem cells are uniformly distributed in the bulbar compartments. ${ }^{59}$ Although cells derived from the 


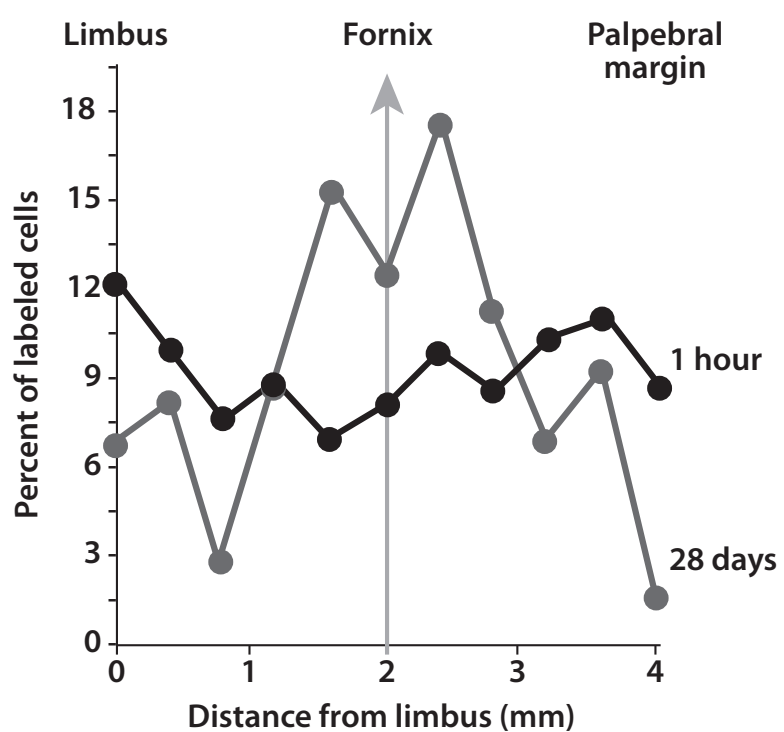

Figure 7. The distribution of $[3 \mathrm{H}]$-thymidine in rat conjunctiva at two time points after a single intraperitoneal injection, 1 hour and 28 days. Label was measured across the full extent of the conjunctiva from lid margin to limbus. One hour after labeling, labeled cells were spread evenly along the basal layer. Superficial cell layers were not labeled. With the passage of time, labeled cells in the fornix became more abundant, while those at the limbus and lid margin decreased. This change in pattern was interpreted as due to the streaming of epithelial cells toward the fornix from both the lid margin and the limbus. (Reprinted from Pe'er J, Zajicek G, Greifner H, Kogan $M^{60}$ with permission of Anat Rec.)

limbus were also studied, no conclusion was drawn about the properties of keratinocytes derived from the lid margin. In an interesting study, Pe'er and colleagues, in the mouse, followed the movement of labeled conjunctival cells after the intraperitoneal injection of tritiated thymidine. ${ }^{60}$ At 1 hour, labeling was spread evenly in the basal layer of the conjunctiva between the limbus and the MCJ. Over a time extending to 28 days, labeling increased in the fornix and declined at both the limbus and the lid margin (Figure 7). From this, they concluded that there was a twocompartment cell renewal system, with progenitor cells for the conjunctiva residing at both the limbus and the MCJ, giving rise to cells that streamed toward the fornix. The streaming velocity of labeled cells was $13.2 \mu \mathrm{m} /$ day from the limbus and $11.6 \mu \mathrm{m} /$ day from the mucocutaneous junction.

The findings of Pe'er et al were confirmed in part, in the rabbit, by Wirtschafter et al, who followed the fate of conjunctival cells, labeled after a single systemic dose of BrdU.61,62 They found an initial focus of label at the MCJ of the lid margin and then, over a period of 2 months, a migration of what they took to be transient amplifying cells, both vertically to the surface of the epithelium and horizontally toward the conjunctival fornix. The migration rate was between 1.68 and $1.84 \mathrm{~mm} /$ day. There was long-term retention of label at the MCJ over the entire observation period. They inferred that conjunctival stem cells were located mainly at the MCJ (Figure 8) and noted that this region, equivalent in their terms to the marginal conjunctiva discussed earlier, was thick in comparison to the adjacent conjunctival epithelium. This is apparent in histological sections of the human lid margin and resembles the niche architecture for stem cells elsewhere in the body, including the corneal limbus, ${ }^{63,64}$ the rete pegs of the skin, ${ }^{65}$ in the bulge region of the hair follicles, ${ }^{66,67}$ and at the base of the crypts in the epithelium of the small intestine. ${ }^{68}$ If these findings are applicable to the human lid margin, then these lid margin stem cells would be wellplaced to maintain a high epithelial cell turnover at this site.

The location of the stem cells for the keratinocytes of skin at the lid margin is not known, but generally, in human skin, stem cells are uniformly distributed in the interfollicular layer of the epidermis, whereas in the human hair follicle, they are segregated to the bulge region of the outer root sheath, below the midpoint of the follicle, as well as in the matrix. ${ }^{69}$

\section{VARIATION OF OSMOLARITY IN THE TEAR COMPARTMENTS}

The aqueous tears are secreted by the lacrimal glands and receive a small additional component from the conjunctiva. ${ }^{70}$ The lacrimal fluid is secreted as an isotonic or slightly hypotonic fluid and the tonicity of the tears is increased by exposure to evaporation during the interblink. ${ }^{71}$ The tear film and menisci are partially refreshed with each blink.

Tear osmolarity is influenced by factors that affect evaporative water loss from the preocular fluid during the interblink, including aqueous tear flow, integrity of the tear film lipid layer, temperature of the preocular tear compartment, size of the palpebral aperture, and duration of the interblink. Environmental factors influencing tear osmolarity include the ambient relative humidity and the air speed

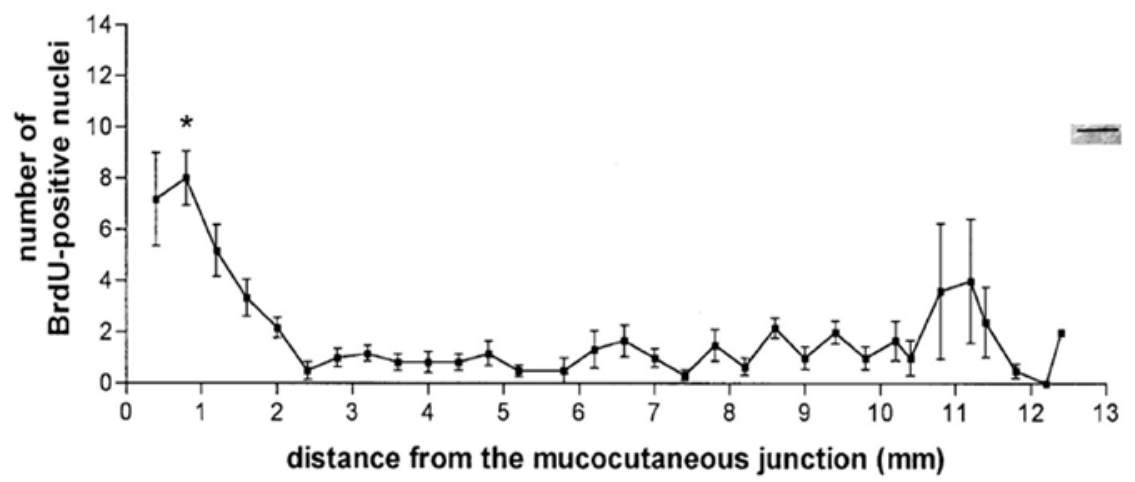

Figure 8. The graph shows the long-term retention of BrdU in rabbit palpebral conjunctiva, from the mucocutaneous junction toward the fornix. After 14 days of daily BrdU administration, animals were allowed to survive for a 2-month wash-out period. Each point represents the mean of at least four eyelid counts. Error bars indicate SEM. Data are significantly different from values at other locations along the palpebral conjunctiva. (Reprinted from Wirtschafter JD, Ketcham JM, Weinstock RJ, et al ${ }^{62}$ with permission of Invest Ophthalmol Vis Sci.) 


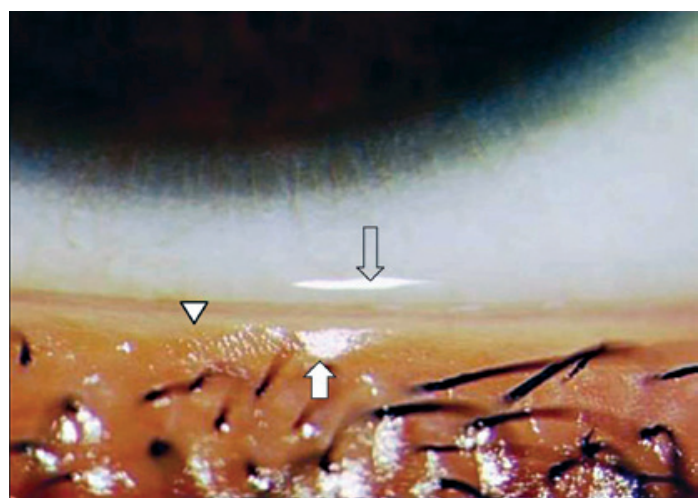

A

B

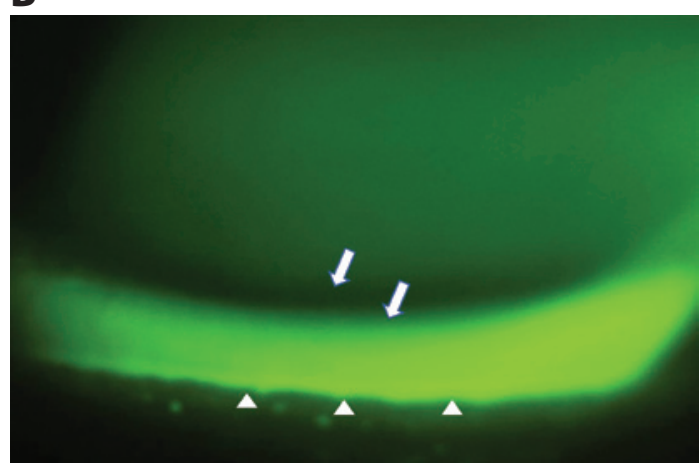

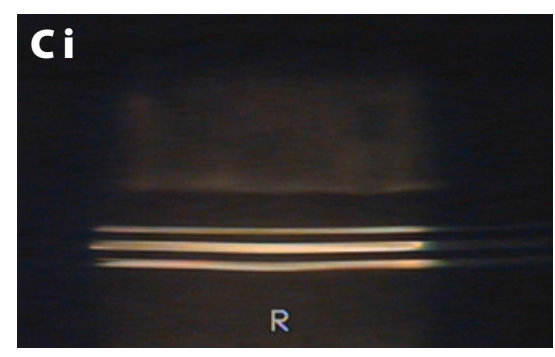
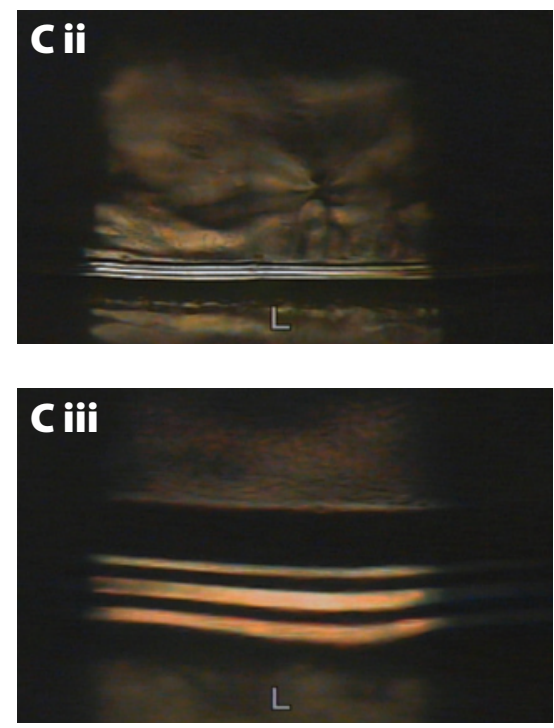

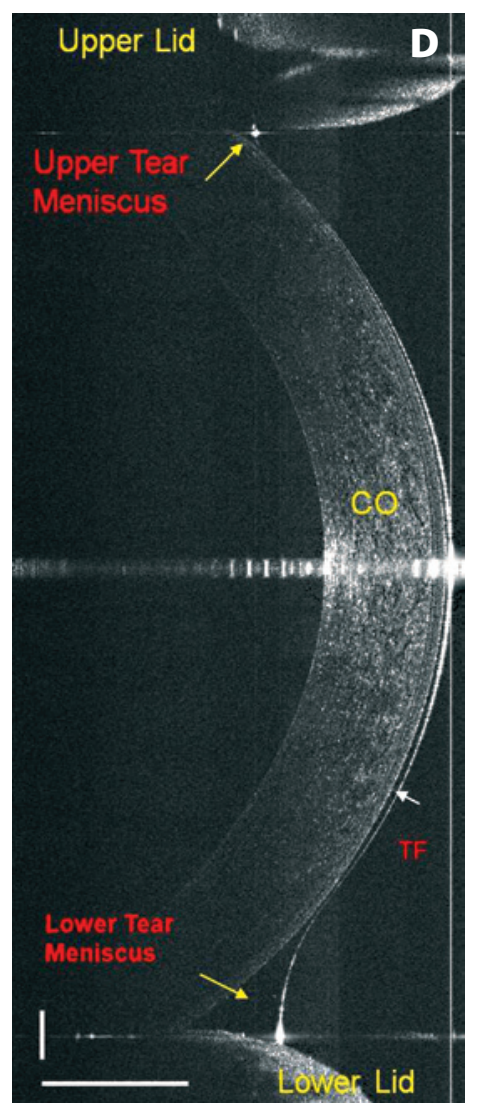

Figure 9. Features of the lower tear meniscus.

A. Slit-lamp photograph showing the tear meniscus in a healthy young adult female. The curvature of the meniscus is indicated by the broad, bright, horizontal streak (open arrow) due to specular reflection from the concave surface of the meniscus. Specular reflection from the lid margin skin demarcates the apex of the meniscus at this site (closed arrow). Note the irregular reflex from the adjacent, occlusal epidermis. The occlusal conjunctiva of the lid margin is seen as a pale narrow strip whose anterior margin is the mucocutaneous junction (arrowhead).

B. Fluorescein-stained, lower tear meniscus in a 32-year-old woman, showing a smooth but slightly scalloped mucocutaneous junction at the peripheral apex of the meniscus (arrowheads) and the "black line," directly above the central apex of the meniscus. The arrows demarcate the peripheral and central boundaries of the black line.

C. Tear meniscus radius of curvature measured by video-meniscometry. i: A normal tear meniscus with a radius of curvature of $0.20 \mathrm{~mm}$. ii: The tear meniscus in a patient with aqueous-deficient dry eye: radius of curvature of $0.11 \mathrm{~mm}$. iii: The tear meniscus in a patient with aqueousdeficient dry eye, expanded after punctal occlusion. Radius of curvature of $0.54 \mathrm{~mm}$. (All illustrations are at approximately the same scale.)

D. Ultra-high resolution optical coherence tomography of the upper and lower tear menisci, imaged with a custom-built ultra-high resolution optical coherence system. The menisci are imaged after the instillation of a single drop of artificial tears (Refresh Liquigel ${ }^{\circledR}$, Allergan Phamaceuticals). The boundaries of the tear menisci were clearly visualized. The lower meniscus is expanded and the anterior radius of curvature increased in comparison to the resting eye. The bars denote to $500 \mu \mathrm{m}$. CO = cornea. TF = tear film. Courtesy of Jianhua Wang, MD, PhD and Victor L. Perez, Bascom Palmer Eye Institute, University of Miami.

over the ocular surface. ${ }^{72}$ In an earlier paper, we proposed a compartmental hypothesis, in which tear osmolarity was conceived to vary between the menisci and the tear film. ${ }^{2}$ It was suggested that the osmolarity of the tears in these two compartments might be affected by differences in their surface area to volume ratio, by variations in the relative humidity of the air overlying different regions of the tear film and menisci, and by the pulsatile nature of flow in the tear menisci. We suggested that the effect of these influences might not be evenly distributed over the exposed ocular surface during the waking day.

It was suggested that the osmolarity of the tear menisci would be lower than that in the tear film and that, therefore, sampling from the lower meniscus might lead to an underestimate of tear film osmolarity. This, in turn, would lead to an underestimate of the level of osmolar stress to which the ocular surface would be exposed in dry eye. Subsequently, mathematical modeling of these interactions has predicted a slightly higher value for tear osmolarity in the normal tear film compared to the menisci and an increasing differential between the two compartments in both aqueous-deficient dry eye (ADDE) and evaporative dry eye. ${ }^{3}$ In the present paper, we suggest that the configuration of the meniscus at the lid margin is an additional factor influencing the distribution of osmolarity within the meniscus itself, giving rise to a further osmolar compartment which has consequences for events at the lid margin.

\section{THE TEAR MENISCI}

The tear menisci consist of two rivers of tears, occupying the angles between the upper and lower lid margins and the globe (Figures 9 and 10). The upper and lower menisci 


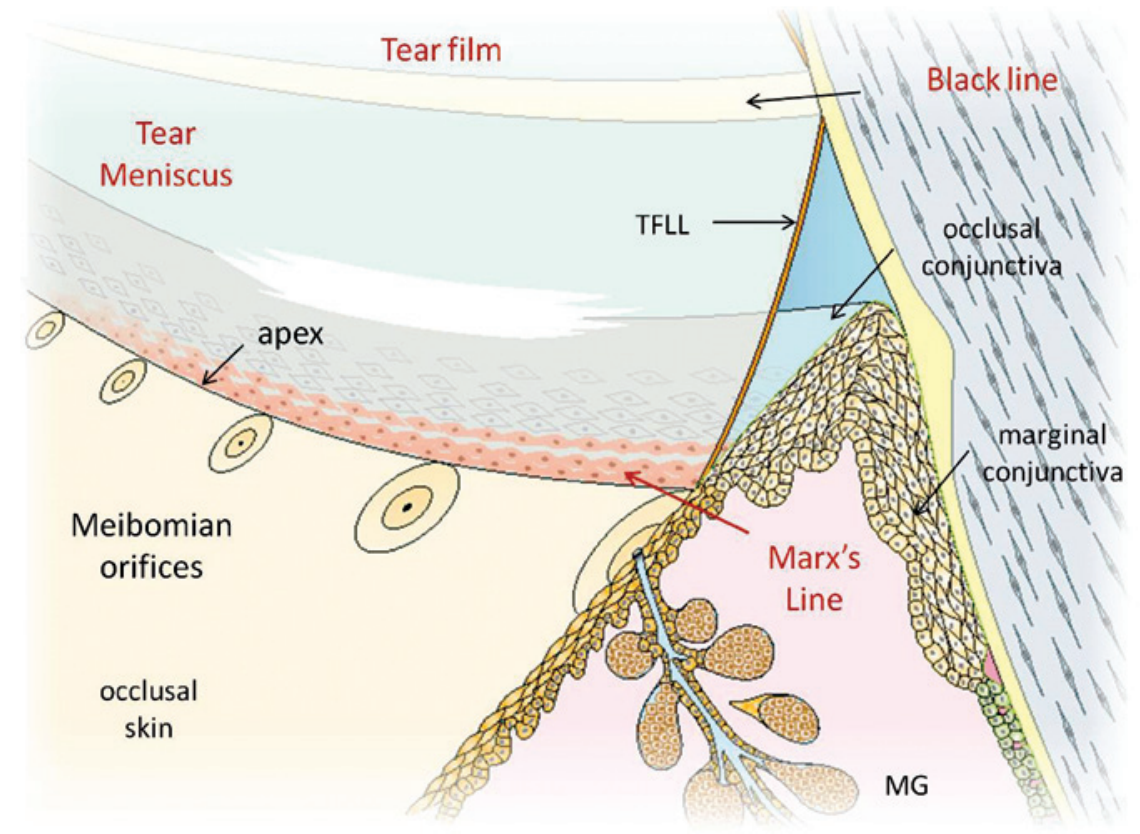

Figure 10. Schematic view of the lower tear meniscus and lid margin. The meniscus overlies and wets both the occlusal part of the marginal mucosa and the adjoining surface of the globe. The peripheral apex of the meniscus is pinned at the mucocutaneous junction (mcj) which forms the boundary between the stratified squamous keratinized epidermis of the occlusal skin of the lid margin, and the stratified squamous nonkeratinized occlusal conjunctiva. It is located directly behind the landmarks that characterize the meibomian gland orifices. The central apex of the meniscus is segregated from the tear film by the "black line." The row of stainable epithelial cells that go to make up Marx's line lie immediately under the apex of the tear meniscus. ber of ways. At high slit-lamp magnification ( $\geq \times 25)$, with diffuse white light, the rough surface of the skin is distinctly different in appearance from that of the pale pink, vascular surface of the occlusal mucosa. The location and configuration of the MCJ may also be observed more precisely by viewing the surface of the lid margin in specular reflection. This can be achieved, for instance, in the lower lid, by using a broad, horizontal slit-beam directed at the lower lid margin at low intensity. As the lid margin is rotated outwards, the highly reflective, oil-covered surface of the lid margin skin is brought into view in specular mode (Figure 9A). With this technique, the surface of the epidermis is readily imaged and shows fine elevations due to the irregular arrangement of the keratinized surface epithelial cells. In particular, the sharp boundary between the lid skin at the MCJ and the apex of the tear meniscus is readily seen. This junctional region can be confirmed after instillation of fluorescein and observation of the stained tear meniscus with use of a blue exciter and yellow barrier filter,

merge temporally at each lateral canthus and blend nasally with the tear lake. They form in the upstroke of the blink and are in continuity with the fluid of the tear film for about 0.1 seconds after the onset of the blink, after which the tear film and menisci become segregated by the formation of the black line, ${ }^{73,74}$ The menisci are roughly wedge-shaped in sagittal section, exhibiting a free, concave, anterior surface in contact with the air, and posterior and peripheral surfaces that bathe and moisten the hydrophilic mucosae of the globe (cornea and bulbar conjunctiva) or occlusal conjunctiva, respectively (Figure 10). The cross-sectional profile of the tear meniscus has been recorded, using slitphotography ${ }^{75}$ and optical coherence microscopy, ${ }^{76,77}$ but while, for the purposes of calculating meniscus volume, it is generally treated as if it formed part of a circle, it is likely to have a more complex shape. The profile is further modified in the presence of epiphora or by drop instillation, either of which increases meniscus volume and the radius of curvature. It will also be influenced by the width of the occlusal zone of the conjunctiva. Because of the resolution of current imaging systems, the detailed configuration of the apical part of the meniscus, as it approaches the MCJ, is not known for certain.

An important feature of the tear meniscus is that its apex, or anterior tip, is pinned at the MCJ, the watershed between the hydrophobic skin and the hydrophilic, occlusal conjunctiva. It is therefore the landmark for the MCJ. This junction and interface can be observed biomicroscopically in a num- matched to facilitate observation of fluorescence. In the latter case, details of the fluorescent anterior border of the tear meniscus become highly visible, although the mucosa is initially obscured by the opaquely fluorescent stream, until fluorescence subsides (Figure 9B).

The sectional contour of the free surface of the meniscus is influenced by the volume of the menisci. Normally this surface is concave. However, with increasing volume, for instance, after the instillation of fluid or in the presence of epiphora, the radius of curvature increases until a point is reached when the surface is first flattened and then becomes convex outwards, prior to brimming over. When the volume of the menisci is low, as in aqueous-deficient dry eye (ADDE), the radius of curvature of the meniscus is reduced. ${ }^{77,78}$ The volume of the tear menisci and, hence, of the total volume of the tears ${ }^{79,80}$ may be inferred indirectly from the radius of curvature of the lower meniscus. ${ }^{80}$

The posterior face of each meniscus is in direct contact with the surface epithelium of the globe (cornea and conjunctiva), while the peripheral face is in contact with that of the occlusal conjunctiva. In general, the wettability of such surfaces is conferred by the surface glycocalyx of these cells, ${ }^{81-83}$ which is composed of their expressed membranespanning mucins. These are the transmembrane mucins, MUC 1, 4 and 16, in case of the cornea, and MUC 1, (2) 4 and 16 in the case of the conjunctiva, ${ }^{84-87}$ which form the epithelial glycocalyx. The role of MUC 16 is of particular interest, as it appears that, at least for the cornea, expression of 
this mucin is characteristic of the mature epithelium ${ }^{88}$ and, in conjunction with galectin-3, determines whether surface epithelial cells are stainable with rose bengal or similar dyes. However, importantly, the expression of mucins by the marginal conjunctiva of the lid has not yet received attention. This may be an important consideration, as the staining characteristics of the epithelium with topical dyes, at the site of Marx's line, differ from those of the conjunctiva proper.

\section{STAINING OF THE OCULAR SURFACE WITH TOPICAL DYES}

\section{A. Historical Aspects}

The use of topical dyes to characterize changes at the ocular surface dates back to 1882, when Pfluger used fluorescein to stain corneal abrasions in the rabbit. ${ }^{89}$ It was later introduced for human clinical use..$^{90,91}$ The use of rose bengal was introduced by Schirmer ${ }^{92}$ and popularized by Sjogren's studies of the disease that was later named after him. Rose bengal is used infrequently now because of the severity of discomfort induced by its instillation. A well-tolerated agent, lissamine green, with similar staining properties, is now available and is used in its stead. Clinically, lissamine green provides information similar to that provided by rose bengal, and it is well tolerated. Great credit is due to Mogens Stig Norn, who established the staining properties of fluorescein, rose bengal, lissamine green, and other dyes at the surface of the eye, in a comprehensive series of studies between 1962 and 1972. ${ }^{29,93-101}$ Other studies of relevance are those conducted by Marx and Maurice, and by Tseng and Argueso and colleagues. 6,88,102-105

\section{B. Rose Bengal and Lissamine Green}

Rose bengal is a hydroxyxanthine dye differing from fluorescein by the substitution of 4 chloride and 4 iodide atoms into the basic molecule. In the past, it has been available commercially as a sterile $1 \%$ solution or in the form of impregnated strips. The degree of staining of pathological lesions by rose bengal is dose-dependent. ${ }^{106}$ Thus, if drop size or concentration is reduced to minimize stinging, the amount of staining is also reduced. Instillation of dye from an impregnated strip is better tolerated than a drop.

When rose bengal is used clinically at the slit-lamp, staining of the ocular surface may be observed in white light, or, alternatively, with use of a red-free absorption filter to enhance visibility. With white light, at low magnification, stained corneal epithelial cells (eg, in dry eye patients) are seen as red dots. At higher magnification, these stained cells are seen as straight-sided, polygonal cells with some uptake of dye into the cytoplasm, but concentrated in the cell nuclei. This staining pattern has been demonstrated convincingly by Tabery (Figure 11). ${ }^{107-109}$ The staining characteristics of rose bengal in the healthy young eye with an intact epithelium are similar to those of fluorescein, as neither dye stains the healthy epithelium. There are exceptions to this which relate to the presence of chance degenerations of the conjunctiva such as pinguecula, or elevated regions such as the caruncle, which will take stain. Scattered, sparse staining also occurs in older age groups ${ }^{29}$ and has been considered to demonstrate normal desquamation.

The classical observations of Norn suggested that rose bengal stains dead and degenerate surface epithelial cells, both at the ocular surface or within mucus strands in the lower fornix. ${ }^{106}$ Staining of corneal punctate epithelial erosions is unusual, but staining of conjunctival punctate epithelial erosions persists without visible decrease or diffusion into the deeper layers of the epithelium for many minutes.

Tseng and associates have studied the in vitro characteristics of rose bengal staining in some detail in rabbit corneal epithelial culture, using a fluorescence microscope with an exciter filter transmitting between 546 and $580 \mathrm{~nm}$ and a barrier filter absorbing at $>590 \mathrm{~nm} \cdot{ }^{103,104}$ They showed a rapid uptake of dye into confluent cultures of rabbit corneal epithelial cells within seconds of exposure, leading to staining, predominantly of cell nuclei but also of other organelles. They observed further that rose bengal uptake could be completely blocked if cells were precoated with any of porcine stomach mucin, bovine serum albumin or carboxymethyl-cellulose. Since, in the normal young human cornea, the surface epithelial cells do not take up rose bengal stain, they concluded that, at the healthy ocular surface in vivo, the surface epithelial cells are protected by tear components, such as mucin or albumin. Their comments referred only to corneal epithelial cells, but, by inference, were relevant to the ocular surface as a whole. They therefore attributed staining by rose bengal in the presence of ocular surface pathology to a "dysfunctional mucous layer." Alternatively, or in addition, they suggested that the apical, epithelial cell "mucous layer" (which, although not 


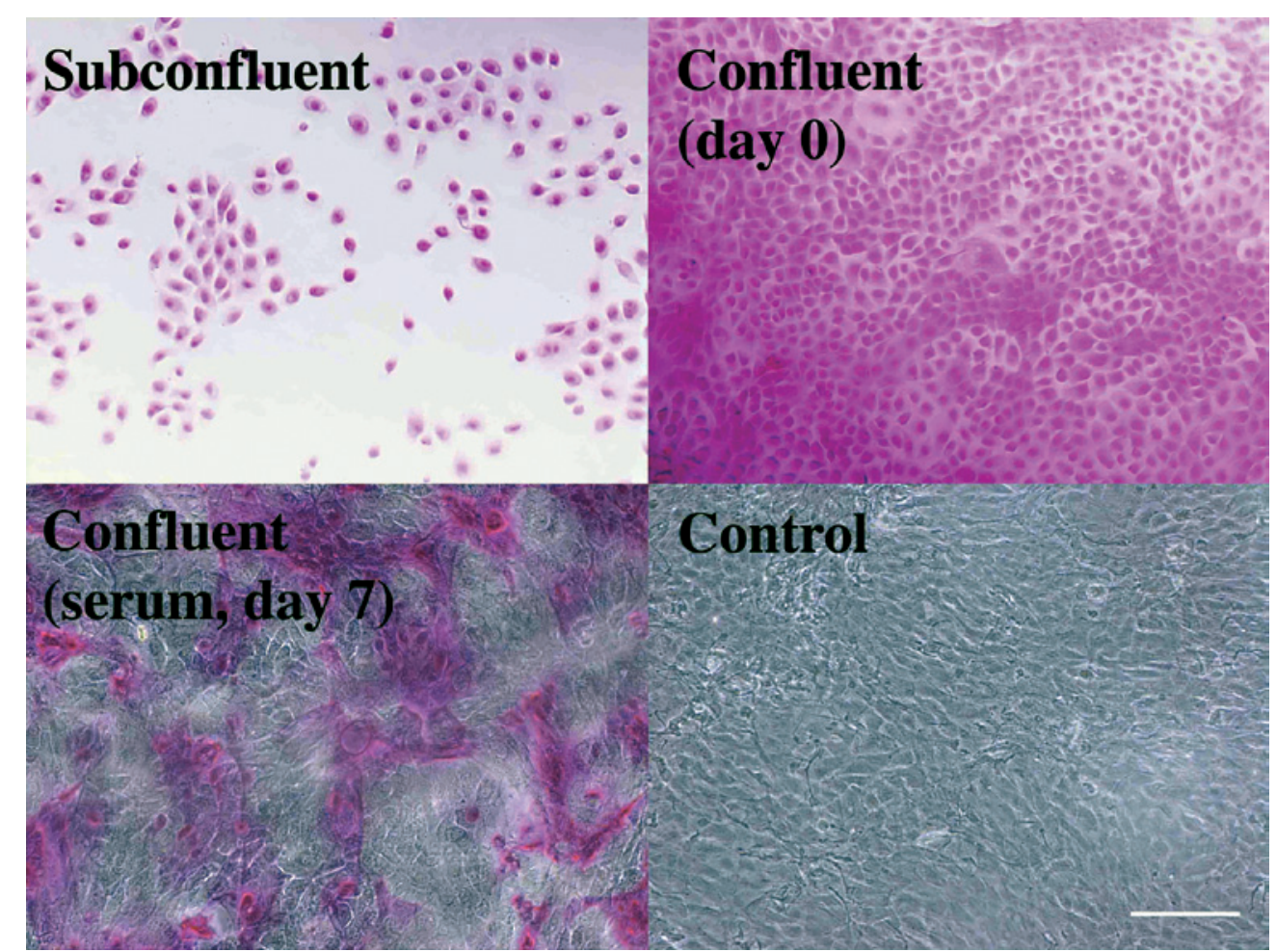

Figure 12. Rose bengal staining of human corneal limbal epithelial cells (HCLE) at different stages during culture. There is rapid uptake of dye into both subconfluent and confluent HCLE cells grown in serum-free medium, with staining stronger in the nuclei than in the cytoplasm. After culture in medium containing $10 \%$ calf serum, by 7 days, islands of stratified, confluent cells appear which resist penetration by rose bengal. Control micrographs are phase-contrast images of unstained cells, grown in serum for 7 days. (Reprinted from Argueso P, Tisdale A, Spurr-Michaud S, et al ${ }^{88}$ with permission of Invest Ophthalmol Vis Sci.) tribute to the development of squamous metaplasia.

Subsequently, it was recognized that the epitope stained by $\mathrm{H} 185$ was a component of a newly discovered membrane-spanning mucin, MUC 16,114 and it was therefore apparent that not only was MUC 16 a component of the normal, apical, corneal epithelial glycocalyx, ${ }^{86,87,115}$ but that rose bengal staining was associated with an absence of expression. Argueso et al resolved this issue by demonstrating that protection from staining by rose bengal depended on the level of maturation of surface cells and the expression of a full complement of glycocalyx mucins. ${ }^{88,116,117}$ They demonstrated that the uptake of rose bengal dye by corneal epithelial cells in culture was dependent on the state of differentiation of these cells and the expression of

stated, would correspond to its glycocalyx) might play the same role in excluding dye entry into surface epithelial cells. In view of the punctate nature of rose bengal staining, the latter explanation, referable to individual epithelial cells, was more logical, since protection by a tear component alone, coating surface cells, would be expected to provide a diffuse protection from stain and would not account for a punctate epitheliopathy.

An explanation for the above-described findings and for the staining characteristics of rose bengal has since been revealed by research directed at the glycocalyx of surface epithelial cells. Early studies using the monoclonal antibody H185, which recognizes a carbohydrate epitope expressed by a normal glycocalyx mucin, showed that the biosynthesis and/or glycosylation of this mucin on the ocular surface is altered in patients with superior limbic keratoconjunctivitis ${ }^{110}$ and non-Sjogren ADDE. ${ }^{110-112}$ In a study by Danjo et al, a positive correlation was found between decreased $\mathrm{H} 185$ antibody binding to apical conjunctival epithelial cells and the severity of rose bengal staining scores in patients with dry eye. ${ }^{12}$ In another study of ADDE patients, Pflugfelder et al found a positive correlation between decreased expression of a transmembrane glycocalyx mucin in conjunctival impression cytology specimens and increased rose bengal staining. ${ }^{113}$ They suggested that membrane-spanning mucins are necessary for the normal differentiation of ocular surface epithelia and that lack of this class of mucins may con- a specific, membrane-associated, mucin glycoprotein..$^{88}$ Human corneal limbal epithelial cells grown to confluence in serum-free conditions and expressing MUC1 but not MUC 16 showed cytoplasmic and nuclear staining with rose bengal after 5 minutes incubation with rose bengal, $0.1 \%$ (Figure 12). At this stage, their culture model resembled that studied by Feenstra and Tseng. ${ }^{103}$ When cultures were switched to DMEM/F12, to promote differentiation, islands of stratified epithelium appeared that expressed MUC 16 (and T-antigen) at their apical surfaces and excluded staining with rose bengal (Figure $12 \mathrm{C}$ and $\mathrm{D}$ ).

Further studies using immortalized human corneal (HCLE) and conjunctival (HCjE) cells have shown that galectin-3, interacting with MUC1 and MUC 16 within the apical glycocalyx, plays a key additional role in the barrier properties of the epithelium. ${ }^{116}$ Inhibition of galectin-3 binding to mucin in these studies resulted in increased rose bengal staining, and galectin- 3 was therefore presumed to cooperate in the exclusion of rose bengal.

In recent years, lissamine green has replaced rose bengal in clinical use. For practical purposes, and notwithstanding some small differences in mode of action at the cellular lev$\mathrm{el},{ }^{9}$ lissamine green may be assumed to offer the same opportunities and interpretations as staining with rose bengal. ${ }^{19,20}$

\section{Fluorescein Sodium}

Fluorescein is a water-soluble, anionic dye introduced 
by Ehrlich in $1882^{118}$ for the staining of tissues and cells in the living state. To examine the integrity of the ocular surface after instillation of fluorescein dye at a suitable concentration, the eye is examined with the slit-lamp microscope and observed using a blue exciter source (eg, "cobalt" light), covering the excitation range of fluorescein, $485-510 \mathrm{~nm}$. In order to observe and record this fluorescence effectively, the eye must be viewed through a yellow filter transmitting over the emission range of fluorescein (515-565 nm [eg, a Kodak Wratten 12 filter]). Fluorescein at high concentration, eg, $1 \%$, is poorly fluorescent, due to self-quenching. However, at lower concentrations, eg, $0.1 \%$, it is highly fluorescent (Figure 13). To achieve a high fluorescence intensity and maximum contrast at the slit-lamp, matched bandpass filters can be used, transmitting at a $\lambda$ max of $491 \mathrm{~nm}$ for the exciter and $517 \mathrm{~nm}$ for the barrier filter, the emission peak of fluorescein. However, for practical purposes and reasons of cost, it is satisfactory to use absorption filters. In clinical use, these also have the advantage of transmitting red light and providing a background that can guide the observer to the clinical target.

Unless otherwise stated here, references to clinical staining with fluorescein refer to the observation of fluorescence using a blue exciter filter while observing through a yellow or blue-free absorption filter (see below). ${ }^{119-121}$ At the level of magnification used in slit-lamp microscopy, fluorescein instilled to produce a fluorescent tear film does not usually stain the healthy surface epithelium of either the cornea or the conjunctiva proper. This has been interpreted to mean that the dye cannot diffuse across healthy epithelial cell membranes or negotiate the tight junctions between surface epithelial cells. It is usually stated that fluorescein requires a loss of surface epithelial cells or breakdown of these tight junctions in order to enter and visibly stain the corneal epithelium. Epithelial cell shedding is a continuous event at the surface of the eye, implying that tight junctions are formed between contiguous epithelial cells underlying surface cells that are about to be shed.

Wilson has provided evidence, in the rabbit, to suggest that corneal epithelial cells are intrinsically stainable by fluorescein dye. ${ }^{122}$ This is in keeping with the observations of Feenstra and Tseng, who showed that fluorescein dye can diffuse into rabbit corneal epithelial cells in culture, although to a much lesser degree than rose bengal. ${ }^{103}$ It also fits in with the observations of Tabery, using fluorescein dye instilled at high concentration, that fluorescein is taken up by (or "adheres" to) the same diseased corneal epithelial cells as those stained by rose bengal. ${ }^{107,109,123}$ The caveat should be accepted that these observations may be subject to the same interpretation as that offered by Argueso et al in relation to rose bengal staining of cultured corneal epithelial cells. ${ }^{88}$ Thus, staining under cell culture conditions may reflect the undifferentiated state of the cultured cells. In this respect, the staining of Marx's line, not only by rose bengal and lissamine green but also by fluorescein, is in keeping with this view. It is notable that, in the absence of disease, fluorescein does not usually stain other regions of the ocular surface epithelia, except in an occasional and scattered manner. ${ }^{100}$

\section{MARX'S LINE}

\section{A. Background}

Marx, using a 5\% rose bengal solution, demonstrated the presence of a narrow line of epithelial staining, extending for the full length of each lid margin in normal lids of subjects of both sexes. The line was stainable from infancy into adult life and could be demonstrated with other acidic (anionic) dyes such as eosin (10\%) and water blue or nigrosin (both $5 \%)^{6,7}$ (Figure 14A). Marx placed the location of this line at the junction of the palpebral conjunctiva and the keratinized skin, ie, at the MCJ, and Norn, using triple staining with fluorescein, lissamine green B and Sudan III, observed the line to lie behind the "ampullae" of the meibomian orifices. In his 1985 paper, Norn illustrates it to extend from the MCJ to the posterior lid margin, which is broader than that normally encountered in the young healthy lid. ${ }^{29}$ Various other authors have identified the stained surface cells to lie immediately behind the MCJ and therefore to represent staining of a few rows of occlusal conjunctival cells adjoining the junction. Our own observations concur with this, and it is apparent that in young normal lids, there is a zone of unstained cells between the posterior edge of the stained line and the posterior lid margin. In general, there is more staining nasally, where the line is thicker, than temporally, and it is apparent that the entire conjunctival sac is surrounded by this stainable row of cells that lie directly behind the MCJ. ${ }^{6}$ Marx concluded that the affected cells were in some way involved in the retention of tears within the menisci. He demonstrated directly, by examining cells 

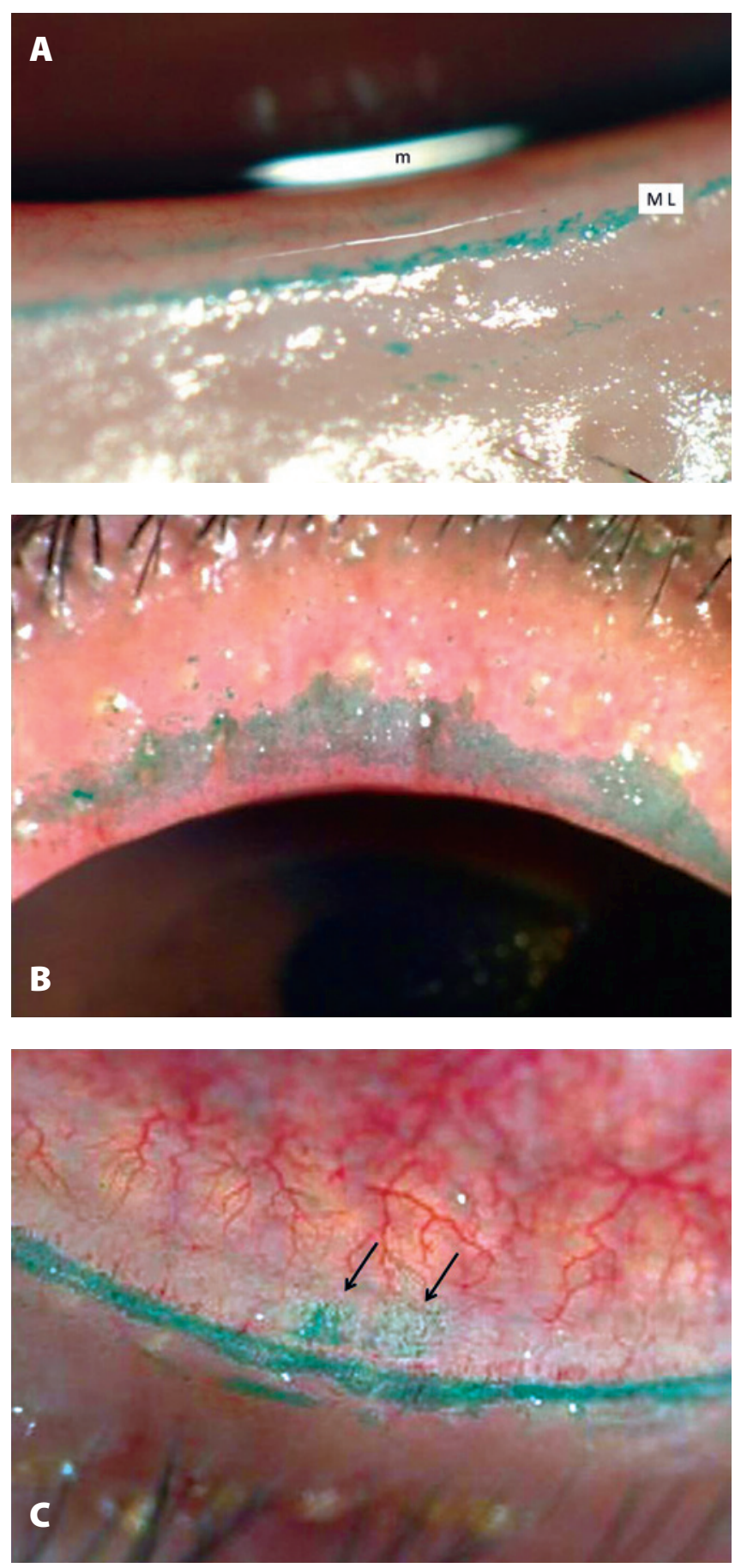

Figure 14. Clinical pictures of Marx's line, stained with lissamine green.

A. Marx's line [ML] of the lower lid of a 24-year-old woman, stained with lissamine green. The occlusal mucosa lies directly behind. The lid is slightly everted, so that the tear meniscus, indicated by a broad, bright specular reflex $(\mathrm{m})$, is drawn slightly backwards.

B. Marx's line of the upper lid, stained with lissamine green, in an 87-year-old woman with mild aqueous-deficient dry eye, showing in addition, combined anterior blepharitis and advanced meibomian gland dysfunction (MGD). Marx's line is markedly broadened and is peaked forward to involve some of the meibomian gland orifices.

C. Marx's line of the lower lid, stained with lissamine green in a 62-year-old man with mild MGD and aqueous tear deficiency. The line is broadened in places, opposite the location of two, lid-wiper like patches of epithelial staining (arrows). $\underline{0}$

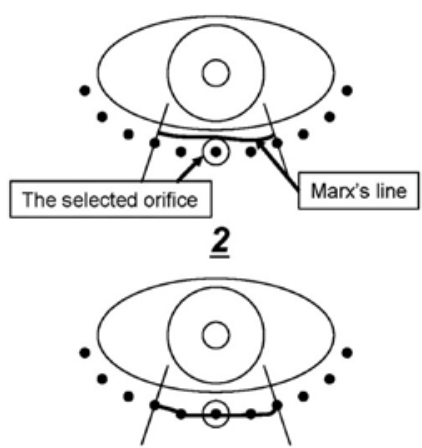

1

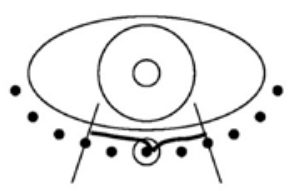

$\underline{3}$

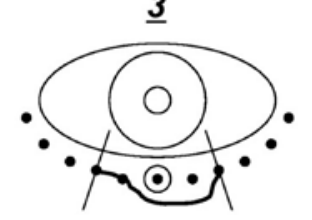

Figure 15. Grading of the location of Marx's line. See text for details. (Reprinted fromYamaguchi M, Kutsuna M, Uno T, et al ${ }^{30}$ with permission of Am J Ophthalmol.)

removed from the stained line microscopically, that the dots of stain were due to the uptake of dye into surface epithelial cells, particularly their nuclei.

At the nasal canthus, Marx's line and the MCJ pass anterior to the puncta, but staining posterior to the puncta and in more complex patterns is also observed. ${ }^{6}$ The staining pattern around the punctum will not be addressed further in this report. Hughes et al have measured the line to be about $100 \mu \mathrm{m}( \pm 90 \mu \mathrm{m} \mathrm{SD})$ in width at the center of the adult lid. ${ }^{19}$ The width varies along the length of the lids. Marx's line has been demonstrated by numerous authors, using rose bengal, lissamine green, fluorescein, and other dyes. ${ }^{6,19,20,29,30,124}$

In younger subjects, the line takes a smoother course, parallel to the posterior margin of the lid, but with increasing age, it shows increasing irregularities of contour, position, and width (Figure 14B and 14C). 19,20,29,30 Yamaguchi et al graded the disposition of the line in normal subjects, using rose bengal and lissamine green in their preliminary studies and fluorescein dye alone in their definitive study $(2 \mu \mathrm{l}$ of $1 \%) .{ }^{30}$ Staining was observed in blue light without the use of a yellow barrier filter. The grading was: $0=\mathrm{ML}$ runs entirely on the conjunctival side of the meibomian orifices; 1 = parts of ML touch the meibomian orifices; $2=$ ML runs through the meibomian orifices; $3=$ ML lies on the skin side of the meibomian orifices (Figure 15). Grading was performed in the inner, central and outer thirds of the lower lid, giving a range of scores for the whole lid of 0-9.

Grading was reasonably consistent between observers. They found that lower lid grades were significantly correlated with upper lid grades and were similar between men and women. With age, the grade score increased, implying that Marx's line (and therefore the MCJ) moves forward with time. Of interest was the finding that the anterior shift began earlier in the nasal and temporal zone of the lid margin than in the center. This is curious, given major differences in functionality of the meibomian glands in these two zones. Korb and Blackie found, in young subjects with normal lids, that expressibility of meibum is far greater in the nasal than the temporal zone, implying that the nasal 
glands are more active. ${ }^{125}$ Of interest, too, is that the initial sign of forward movement is an encroachment on individual meibomian orifices. The relationship between Marx's line and meibomian gland dysfunction is discussed in the article that follows this report. ${ }^{4}$

\section{B. Etiology of Marx's Line}

Marx's line is of interest because it represents a collection of healthy surface epithelial cells that exhibit a staining pattern characteristic of damaged cells. It has also intrigued investigators, who are trying to understand how this unusual cohort of cells could come into being at such a discrete location on the lid margin. It has been suggested that these cells correspond to clusters of parakeratinized cells lying behind the MCJ. ${ }^{10,28}$ As noted earlier, Marx's line can be demonstrated in normal lids of both sexes across the lifespan. Pathologically, it may increase in extent or intensity on either lid and may fuse with the stained lid wiper epitheliopathy of the upper lid or with its counterpart in the lower lid.

The following explanations for Marx's line may be considered.

\section{An Intrinsic Property of the Occlusal Cells of the Mucosa}

It may be proposed that the properties of surface epithelium in the region of Marx's line are specified genetically and are not induced by external stresses. No information in support of this view is available, but Riau et al, in the mouse, identified a transitional zone between the epidermis of the lid margin skin and the adjoining conjunctiva, expressing overlapping biomarkers intermediate between those of the skin and conjunctiva. ${ }^{27}$ Such an intrinsic state would not explain the broadening and migration of Marx's line found in human lid margins with age and in disease states.

\section{Mechanical Trauma}

Some authors have considered the line to be formed by a frictional process involving appositional contact between the lid margins during the blink. ${ }^{25}$ The normal blink involves several events, the most prominent of which is the downstroke of the upper lid, whose free margin comes briefly into contact with that of the lower lid during eye closure, before it rises again in the upstroke. The blink is completed in a matter of about 200 ms. $^{72,126-128}$ During the blink, the lower lid margin moves slightly upwards and nasally to meet the descending lid margin and, in many instances, the puncta are brought into apposition. ${ }^{126,127}$ If the waking day is considered to be 16 hours and the blink interval 5 seconds, then at least 12,000-13,000 blink impacts occur each day in the normal subject. This is undoubtedly a conservative estimate.

A curious aspect of this literature is that, while it might be reasonable to envision that a combination of compression and side-to-side rubbing of the apposed lid margins might contribute to the Marx's line phenomenon, some accounts put forward the idea that the site of natural friction (rubbing) is between the marginal zone of the lids and the bulbar and corneal surfaces. ${ }^{18,19,35}$ As indicated in the account given here, whereas the global part of the marginal mucosa is in contact with the bulbar conjunctiva or cornea at all times, only the most posterior edge of the occlusal conjunctiva comes into contact with the globe. In normal lids, Marx's line is located at the anterior margin of the occlusal conjunctiva. Thus, in the absence of a malposition of the lid margins, such as in entropion, there is normally no expectation of a physical interface between the relevant regions of the lid margins and the globe. The anatomical unlikelihood of contact between the Marx's line zone and the globe has been confirmed directly by Korb and Blackie. ${ }^{124}$ It may be relevant, as noted earlier, that the region of Marx's line is slightly elevated above the plane of the epidermal surface of the occlusal lid margin.

In balance, given the narrow width of Marx's line in youth, it seems unlikely that compression or friction between the apposed lid margins is a primary determinant of the line, although it might contribute to the level of the permeability defect and hence to the intensity of staining.

\section{Desiccation}

Hughes et al, identifying the location of Marx's line as conjunctival, considered the possibility that the line represents a region of epithelial desiccation. ${ }^{19}$ This is a proposition that Norn had already considered and rejected, observing that the meniscus was a river of watery fluid covering the line, which precluded drying. ${ }^{96}$ Hughes et al argued that if rose bengal and lissamine green stain devitalized cells or cells lacking a "mucous coat," then the staining pattern would be consistent with these cells having been damaged by desiccation. ${ }^{19}$ However, they were unable to accept dehydration as the etiology in normal subjects, since, they concluded, these cells are normally covered by a multilayer of mucin, aqueous tears and the tear film lipid layer, of the intact meniscus. For this reason, they discarded desiccation as a cause for Marx's line in normal lids, but offered the concept of drying as a mechanism to explain a later extension and broadening of Marx's line in the presence of blepharitis and in situations where there was anterior migration of Marx's line. In this case, it was suggested that desiccation damage to epithelial cells required the presence of an irregular and/or interrupted tear meniscus. It was not, however, indicated how this might induce desiccation. They suggested that in the case of the anterior migration of Marx's line, there was probably a subtle change in the "locus of contact" between the lid margin and the corneal surface.

Hughes et al concluded that, "Marx's line is a unique zone of squamous cells situated along the marginal conjunctiva, just posterior to the tarsal gland orifices...... that [is] the site of slight but routine friction between the conjunctiva and the corneal surface." 19

\section{A Solute Gradient to Explain Marx's Line: A New Hypothesis}

In the introduction to this review, an account of tear distribution, lid anatomy and the interaction of topical dyes 
with surface epithelia was provided as a background to a novel hypothesis to explain certain physiopathological events at the lid margin, including Marx's line.

Each meniscus is formed in the upstroke of the blink and possesses a peripheral apex, pinned to the MCJ, and a central apex formed at the site of the "black line." The black line is a line of tear film thinning or absence, which appears just central to each meniscus after the blink and segregates the tear film from the menisci. It is visualized in the fluorescein-stained tear film as a nonfluorescent (therefore, black), curved, horizontal line of thinning in an otherwise fluorescent film (Figure 9B). ${ }^{73,74,129}$ According to Miller, the black line appears within 0.1 seconds of the start of the interblink and is, therefore, formed as the menisci form. Its relation to the surface of the globe varies with eye movement and position.

We hypothesize here that evaporative water loss from the tear meniscus creates a gradient of solute across its profile, leading to increased tear molarity at each meniscus apex. This is shown schematically in Figure 16. If we assume initially that evaporation per unit area is uniformly distributed across the concave surface of the meniscus, then it can be predicted that, during the interblink, osmolarity will rise more at each apex, where the meniscus is thinnest. It is proposed that this gradient is sufficient to create a hyperosmolar state at the apices, amplified over many blink cycles, which exceeds any that might be generated elsewhere in the meniscus or in the central tear film. It is suggested that, peripherally, this hyperosmolar region, acting as a "meniscal knife," stresses a narrow band of occlusal surface epithelial conjunctival cells, directly behind the mucocutaneous junction. Centrally, it is suggested that, while a hyperosmolar condition could be generated, the mobility of the globe surface in relation to the apex diffuses and diminishes the osmolar stress imparted to the globe. It was noted earlier that there is a drainage flow in the menisci for roughly the first 2 seconds of the interblink period. ${ }^{127} \mathrm{~A}$ basic premise of this gradient hypothesis is that the rising osmolarity at the apices of each meniscus will not be significantly offset by diffusion of solute away from the apex into the bulk of the meniscus, by mixing during the blink or by advection (flow) during the interblink. In reviewing the balance between those events leading to concentration of solute at the peripheral meniscus apex and those diluting it by diffusion, the following arguments may be considered (Tables 1 and 2):

\section{a. Pinning of the meniscus apex.}

There is a sharp boundary between the wettable occlusal conjunctival epithelium and the nonwettable epidermis at the MCJ. The peripheral meniscus apex is thin and is pinned at the MCJ, a critical difference between this boundary and the central apex, which forms at the black line. The black lines demarcate the menisci and tear film by forming a boundary between the inner perimeter of the menisci and the outer perimeter of the tear film. However, whereas the peripheral apices of the tear menisci are pinned to the occlusal mucosa at the MCJ, the black lines are related to regions of the cornea and conjunctiva, which vary in position during eye movement. Therefore, whatever level of hyperosmolarity is achieved in this region is spread over a wider epithelial area by movement of the globe and would not be expected to influence the staining characteristics of the underlying mucosa.

\section{b. Geometry of the meniscus.}

A shallow profile of the meniscus apex would be expected to exacerbate the concentrating influence of evaporation due to an unfavorable ratio of surface area to volume over this zone compared to the bulk of the meniscus. Very shallow profiles also imply a significantly reduced aqueous fraction at the apex of the meniscus, enhancing the influence 
of mucins, as described below. Given that the pressure gradients required to induce flow in thin films increase dramatically and nonlinearly with reduced film depth, ${ }^{130}$ a shallow profile will also inhibit advection. Although some information is available about the meniscus shape, based on the results of slit-lamp ${ }^{10}$ and confocal assessments, ${ }^{76,77}$ the degree of resolution at the present time is not sufficient to describe the inclination of the surface in the immediate vicinity of the MCJ. Nor have such techniques addressed changes in profile that occur over the full extent of the blink interval.

\section{c. Physicochemical properties of the solute.}

The rate of diffusion of solute away from the meniscus apex is governed by molecular size, shape, and charge. The rate of diffusion of sodium ions in water is rapid, while that of proteins and of macromolecules, such as mucin, is much slower in proportion to their molecular size. Thus, if small ions are concentrated at the meniscus, it is certain that the concentration of larger molecules will build up also, and it is conceivable that molecules the size of gel mucin might be effectively trapped at the apex. If mucin concentration were to be enhanced at the meniscus apex, this would offer a further barrier to the diffusion of small ions at that site.

\section{d. Physicochemical interactions between solutes present in the tears.}

The presence of mucins in the tears would be expected to decrease the rate of diffusion of small ions, such as sodium, by an order of magnitude. ${ }^{131}$ The tears contain soluble mucins, such as MUC 7, mucin fragments, and MUC5AC gel mucin. ${ }^{132}$ Binding of cations, such as sodium, and particularly calcium, to mucin would also alter the distribution of ions within the tear meniscus, leading to a greater concentration at the apex. Their contribution to molarity is less certain.

\section{e. Physicochemical interactions between solutes and the glycocalyx.}

Interactions between tear solutes and the epithelial microvilli and glycocalyx would be expected to obstruct diffusion across the surface of the occlusal epithelium.

The gradient hypothesis put forward here depends for its validity on the interaction of many factors, and these are summarized in Tables 1 and 2. The relative importance of each is not yet known, and exploring such phenomena is the subject of current mathematical modeling. Initial observations from such investigations reveal that a sufficiently shallow meniscal profile will lead to elevated solute concentrations at the meniscal apices, providing that the extent of mixing during the blink is limited, for example, due to the accumulation of mucins and an associated increased viscosity near the mucocutaneous junction. However, for levels of diffusion associated with physiological salts, such as $\mathrm{NaCl}$ in water, ${ }^{133}$ the profile has to be exceptionally shallow for the maintenance of concentration gradients, even after accounting for the accumulation of solute at the meniscal apices over large numbers of blinks. This emphasizes the potential importance of mucins in the gradient hypothesis and, particularly, the possibility of mucin trapped at the meniscus apex. These studies will explore how solute levels at the meniscal apices depend on the biophysical mechanisms that underlie the gradient hypothesis.

\section{Events Predicted by the Gradient Hypothesis During the Interblink}

During the interblink, the exposed tears lose water by evaporation, leading to a rise of osmolarity in the tear film and the menisci, ${ }^{134,135}$ reaching a peak just before the next blink, after which the cycle repeats itself, leading, according to modeling considerations, to a slow rise in molarity with repeated blinks over several hours after waking. ${ }^{3}$ Increasing the blink interval would have the effect of amplifying hyperosmolarity in the tear film and menisci.

Similarly, tear osmolarity during the interblink will be lowest in conditions that reduce evaporative water loss and increase meniscus volume, which occur in situations of high ambient humidity and low wind speed. These might be encountered environmentally or could be created by the wearing of occlusive goggles. They will also occur in the presence of lacrimal obstruction or epiphora, when the meniscus volume will be high and apical profiles steep. Conversely, conditions of low humidity and high wind speed and decreased tear secretion would increase hyperosmolarity in the tear film and meniscus. The solute gradient mechanism proposed here would amplify any increase in tear molarity at the meniscus apex.

\section{An Explanation for Marx's Line Based on the Gradient Hypothesis}

The staining pattern of Marx's line is paradoxical. It is encountered in normal subjects of both sexes and at all ages examined, on lid margins, which in youth exhibit an occlusal mucosa that is entirely wettable, just like the epithelial cells of the conjunctiva proper. On the other hand, the clinical staining properties of the involved cells puts them in the same category as pathologically damaged cells, which stain with dyes in dry eye, punctate epithelial keratitis, and squamous metaplasia. The role of tear hyperosmolarity as the central mechanism damaging the epithelium of the ocular surface in dry eye disease ${ }^{136-138}$ was emphasized in the 2007 International Dry Eye Workshop (DEWS), ${ }^{1}$ and early studies reported a significant positive correlation between tear film hyperosmolarity and rose bengal staining in patients with dry eye disease. ${ }^{139}$ It is generally accepted that the relationship is cause-and-effect. It would therefore be reasonable to infer that the staining pattern of Marx's line could be brought about by tear hyperosmolarity.

A key prediction of the gradient hypothesis is that a narrow band of cells within the occlusal region of the marginal mucosa, immediately behind the MCJ, is exposed to hyperosmolar stress, which evolves progressively during the course of the waking day. We hypothesize here that this stimulates a high epithelial turnover at the site of Marx's line and a failure of the surface cells in this region to mature. The presence of conjunctival stem cells at the lid margin, if this is 
Table 1. Factors that may act to reduce solute concentrations at the meniscal apex

\section{Diffusion}

- Transports salts and other solutes down concentration gradients and hence away from the meniscal apex.

\section{Advection}

- Fluid flows have the potential to advect high solute concentrations away from the apical meniscus. For example, tear drainage in the initial interblink may draw fluid from the meniscal apices and reduce the accumulation of solute concentrations. The influence and importance of such flows is difficult to determine a priori; however, they are likely to be lessened by viscosity increases associated with the presence of mucin, the influences of a shallow meniscal profile and the pinned contact line on the lid margin; see Table 2.

\section{Mixing}

- During a blink, apical meniscal fluid may mix with fluid from other regions of the tear film. This effect is likely to be lessened by the viscosity of mucins in the apical meniscus. In particular, mixing essentially does not occur in highly viscous fluids subjected to periodic forcing, as elegantly demonstrated by GI Taylor. ${ }^{145}$ Non-Newtonian behaviors could violate the physical principles underlying such observations, ${ }^{146}$ but the likely very low Reynolds number in fluids at the meniscal apex suggest that viscous effects may dominate.

confirmed in the human, is therefore of interest and would be important to meet the demands imposed by this hyperosmolar stress. The biological state of the surface cells of Marx's line would correspond to the condition of confluent human corneal limbal epithelial cells of Argueso et al prior to their transfer to serum. ${ }^{88}$ These cells, like the rabbit corneal epithelial cells studied by Feenstra and Tseng, ${ }^{103}$ readily took up rose bengal stain. Argueso et al interpreted this to be due to incomplete cell differentiation, with the lack of expression of the glycocalyx mucin MUC 16 and of galectin-3. ${ }^{88,116}$

In recognition of this, we propose that the epithelial staining of Marx's line cells is the consequence of a rapid epithelial cell turnover and incomplete terminal differentiation, including, at least, a deficient expression of MUC16. It is also possible that this immaturity has other features, including deficiencies in the incorporation of galectin- 3 in the glycocalyx or in the expression of cell junctional proteins. It is predicted that a full complement of mucins would be expressed in the neighboring, nonstaining cells of the marginal mucosa. At the time of this writing, the distribution of the glycocalyx mucins of the tarsal conjunctiva, let alone those of the occlusal conjunctiva, has not yet been reported, and this is an area of current research. Although the wettability of the conjunctiva normally depends on the presence of a full complement of transmembrane glycocalyx mucins, it is possible that surface cells would still be wettable with an incomplete glycocalyx, aided by the presence of residual
Table 2. Factors that may act to increase solute concentrations at the meniscal apex

\section{Evaporation}

- Removes fluid, shallowing the meniscal profile and increasing solute concentrations.

\section{Mucin}

- Reduces solute diffusivity in a concentration-dependant manner. ${ }^{131}$

- Reduces salt transport by cationic binding ${ }^{141,142}$ which, in the case of $\mathrm{Ca}++$, also induces a more densely tangled mucin gel, ${ }^{142}$ which is anticipated to be of even higher viscosity.

- Increases viscosity, limiting fluid flow. This may reduce the concentrating influence of evaporation-induced capillary flow (below), but will lessen the influence of advection and blink-induced mixing, which could otherwise decrease solute concentration.

\section{Evaporation-induced capillary flow}

- By analogy to the mechanism that causes coffee stains to form on a surface, ${ }^{143}$ evaporation near the contact line can drive flow into the meniscal apices, compensating for evaporative fluid loss. The fluid introduced into the apices in this manner also evaporates, leading to a net movement of solute into the mucocutaneous junction.

\section{Shallow meniscal profile}

- Exaggerates the influence of evaporation at the meniscus apex due to the high surface area-volume ratio here.

- The pressure gradients required to induce a given flux within a thin film increase dramatically and non-linearly as the film thins. ${ }^{143}$ Thus, advection is likely to be substantially reduced as the profile shallows.

\section{Physicochemical properties of the solute}

- Molecular diffusion decreases with increased molecular size, as epitomized by the Stokes-Einstein relation ${ }^{144}$; thus, large molecules may accumulate in the meniscus apex, directly behind the mucocutaneous junction.

\section{Glycocalyx}

- Interactions and entanglements between tear film and membrane-bound mucins would be expected to obstruct solute transport and fluid flow.

\section{Pinned contact line at the mucocutaneous junction}

- At the mucocutaneous junction, the pinned contact line forces fluid to be stationary at this site, acting to reduce fluid flows and mixing in this region. Pinning also contributes to the mechanism for evaporation-induced capillary flow in the coffee stain model. ${ }^{143}$

\section{Accumulation}

- Even if these factors induce only a small increase in solute concentrations over a single interblink, a substantial solute concentration may still develop over the course of an extremely large number of blinks during the day. When sufficiently high concentration gradients develop, diffusive transport away from the meniscal apex would be upregulated to eventually suffice for dynamic equilibrium. 
glycocalyx mucins and the effect of tear mucins, such as the soluble mucin MUC 7 of lacrimal origin or MUC 5AC gel mucin gel of goblet cell origin. It has been noted that, although the staining mechanism for rose bengal and lissamine green on the one hand and fluorescein on the other may be quantitatively different, each of these dyes stains Marx's line. This is in keeping with earlier observations reported in this paper that each dye may stain immature or damaged corneal epithelial cells, but to a different degree.

While $\mathrm{Na}+$ ions make the greatest contribution to tear hyperosmolarity in dry eye, the concentration of other ions, normally present at low concentration, is also altered. It is therefore relevant to the mechanism of dry eye in general and to the staining mechanism of rose bengal that, in the experiments of Argueso et al, an increase in $\mathrm{Ca}^{2+}$ concentration in the culture medium, and to a lesser extent $\mathrm{Mg}^{2+}$, also decreased the barrier to rose bengal staining. ${ }^{88}$ They argued that the effects of these divalent cations on epithelial cell barrier function might be independent of those due to hyperosmolarity alone. The gradient mechanism proposed here would affect all solute molecules in the tear meniscus.

\section{E. Potential Modifiers of Marx's Line}

If the gradient hypothesis explains the mechanism and location of Marx's line, then some predictions can be made and tested. The hypothesis predicts that the primary driver leading to the stainability of Marx's line cells is evaporative water loss from the tear meniscus. Anything that would reduce this loss should be associated with a decrease in Marx' line staining, and anything increasing it should augment its intensity and encourage its evolution. Thus, it can be predicted that in normal subjects, prolonged wearing of occlusive goggles would result in the disappearance of Marx's line as a result of the reduced evaporative water loss from the tear meniscus. Similarly, a full meniscus arising as a result of nasolacrimal obstruction would be predicted to produce a fainter Marx's line.

A parallel prediction is that Marx's line would not be detectable in the presence of a longstanding complete ptosis induced, for instance, by complete III nerve palsy, since no meniscus would be present and no evaporation would occur. In general, it can be predicted that the intensity of Marx's line will be reduced after a period of prolonged lid closure from any cause, including overnight sleep or prolonged patching. The time required to lose a stainable Marx's line would be expected to depend on the turnover of epithelial cells at the lid margin.

It has been noted by Korb and colleagues (personal communication) that the stainability of Marx's line is poor in infants (Korb D, personal communication, cited with permission). This is, at first glance, surprising, since the blink rate in the very young has been reported to be extraordinarily infrequent, giving rise to the expectation of greater desiccation at the ocular surface. Lawrenson et al found the blink rate in a group of neonates and infants (mean age: $27.5 \pm 15$ sd weeks) to be $3.6 \pm 0.3$ blinks min $^{1}$, with a mean interblink time of $21.6 \pm 2.8 \mathrm{~s} .{ }^{140}$ The slowest blink rate, in the 0-17 week age group, was an average of 2 blinks $\min ^{1}$. The blink rate increased with increasing age. However, they concluded, on the basis of interferometric assessments of the tear film lipid layer that the lack of dry eyes in the infant, despite infrequent blinking, was due to the presence of a thicker tear film lipid layer than in the adult and, possibly, a smaller palpebral aperture. This explanation would also apply to the induction of Marx's line, although a high restorative ability of lid margin stem cells could be an additional factor at this age.

Conversely, it would be predicted that the intensity of staining of Marx's line would be greater in individuals working in environments of low ambient humidity or in high wind speed. This would include working in or travelling in air-conditioned environments. Further, it is predicted that Marx's line would be amplified by conditions enhancing desiccation at the lid margin, such as VII cranial nerve palsy, where there is incomplete lid closure. It may be noted in passing that if Marx's line is demonstrable in the presence of incomplete lid closure, this will demolish any hypothesis that is dependent on physical trauma during the blink. These possibilities are currently under study.

\section{SUMMARY AND CONCLUSIONS}

We have proposed a novel, solute gradient mechanism to explain the occurrence of Marx's line. We hypothesize that evaporation from the tear meniscus, together with its peculiar geometry and the composition of the tears, results, after many iterations of the interblink, in an increase in solute concentration at the peripheral apex of each meniscus. Increased solute concentration, in the form of hyperosmolarity, is put forward as a source of tissue stress delivered to the mucosa immediately behind the MCJ. This is considered to stimulate an increase in cell turnover behind the MCJ, leading to incomplete maturation of the epithelium and a deficient surface glycocalyx. Experimental studies have indicated that the absence of certain glycocalyx components such as MUC-16 and galectin-3 removes a key barrier to the penetration of dyes into the epithelium. We propose that such a deficiency is the basis of the staining properties of Marx's line. Continued stress at the level of the MCJ, acting over a lifetime, is proposed to explain its anterior migration with age. The hypothesis proposed here is capable of experimental proof, and the propositions put forward are currently under investigation. In an accompanying article in this journal, ${ }^{4}$ we address the further implications of this hypothesis for progressive disease at the lid margin, including meibomian gland dysfunction.

\section{REFERENCES}

1. (No authors listed).. 2007 Report of the International Dry Eye WorkShop (DEWS). Ocul Surf 2007;5:69-204

2. Bron AJ, Tiffany JM, Yokoi N, Gouveia SM. Using osmolarity to diagnose dry eye: A compartmental hypothesis and a review of our assumptions. Adv Exp Med Biol 2002;506(Pt B):1087-95

3. Gaffney EA, Tiffany JM, Yokoi N, Bron AJ. A mass and solute balance model for tear volume and osmolarity in the normal and the dry eye. Prog Retin Eye Res 2009;29:59-78 
4. Bron AJ, Yokoi N, Gaffney EA, Tiffany JM. A solute gradient in the tear meniscus. II. Implications for lid margin disease, including meibomian gland dysfunction. Ocul Surf 2011;9(2):92-7

5. Hykin PG, Bron AJ. Age-related morphological changes in lid margin and meibomian gland anatomy. Cornea 1992;11:334-42

6. Marx E. Uber vitale Farbung des Auges und der Augenlieder. I. Uber Anatomie, Physiologie und Pathologie des Augenlidrandes und der Tranenpunkte. Graefe's Arch Ophthalmol 1924; 114:465-82

7. Marx E. Uber vitale Farbung des Auges und der Augenlieder. I. Uber Anatomie, Physiologie und Pathologie des Augenlidrandes und der Tranenpunkte. Graefe's Arch Ophthalmol 1926;116:114-25

8. Wolff E. The muco-cutaneous junction of the lid margin and the distribution of the tear fluid. Trans Ophthalmol Soc U K 1946;66:291-308

9. Munger BL, Halata Z. The sensorineural apparatus of the human eyelid. Am J Anat 1984;170:181-204

10. Knop E, Korb DR, Blackie CA, Knop N. The lid margin is an underestimated structure for preservation of ocular surface health and development of dry eye disease. Dev Ophthalmol 2010;45:108-22

11. Pult H, Korb DR, Blackie C, Knop E. About vital staining of the eye and eyelids. I. The anatomy, physiology, and pathology of the eyelid margins and the lacrimal puncta by E. Marx. Optom Vis Sci 2010;87:718-24

12. Halata Z, Munger BL. The sensory innervation of primate eyelid. Anat Rec 1980;198:657-70

13. Bron AJ, Tripathi RC, Tripathi BJ: Wolff's Anatomy of the Eye and Orbit. London, Weinheim, New York, Tokyo, Melborne, Madras: Chapman \& Hall Medical, 1997, ed 8

14. Virchow H: Mikroskopische Anatomie der ausseren Augenhaut und des Lidapparatus, in Graefe-Saemisch Handbuch der gesamten Augenheilkunde, vol 1. Leipzig, W. Engelmann, 1910, p 431

15. Wulc AE, Dryden RM, Khatchaturian T. Where is the gray line? Arch Ophthalmol 1987;105:1092-8

16. Mackie IA. Riolans muscle: action and indications for botulinum toxin injection. Eye 2000;14:347-52

17. Collin JRO: A manual of systematic eyelid surgery. London and Edinburgh, Churchill Livingstone, 1983.

18. ParsonsJH:Thepathologyoftheeye, Vol1.Hodder\&Stoughton,London, 1904

19. Hughes C, Hamilton L, Doughty MJ. A quantitative assessment of the location and width of Marx's line along the marginal zone of the human eyelid. Optom Vis Sci 2003;80:564-72

20. Doughty MJ, Naase T, Donald C, et al. Visualisation of „Marx's line“ along the marginal eyelid conjunctiva of human subjects with lissamine green dye. Ophthalmic Physiol Opt 2004;24:1-7

21. Bron AJ, Tiffany JM, Gouveia SM, et al. Functional aspects of the tear film lipid layer. Exp Eye Res 2004;78:347-60

22. Jester JV, Nicolaides N, Smith RE. Meibomian gland studies: histologic and ultrastructural investigations. Invest Ophthalmol Vis Sci 1981;20:537-47

23. Jester JV, Nicolaides N, Smith RE. Meibomian gland dysfunction. I. Keratin protein expression in normal human and rabbit meibomian glands Invest Ophthalmol Vis Sci 1989;30:927-35

24. Milz S, Neufang J, Higashiyama I, et al. An immunohistochemical study of the extracellular matrix of the tarsal plate in the upper eyelid in human beings. J Anat 2005;206:37-45

25. Kozak I, Bron AJ, Kucharova K, et al. Morphologic and volumetric studies of the meibomian glands in elderly human eyelids. Cornea 2007;26:610-4

26. McDonald JE. Surface phenomena of the tear film. Am J Ophthalmol 1969;67:56-64

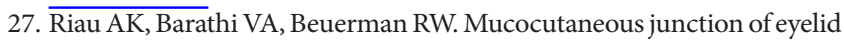
and lip: a study of the transition zone using epithelial cell markers. Curr Eye Res 2008;33:912-22

28. Knop E, Knop N, Zhivov A, et al. The lid wiper and muco-cutaneous junction anatomy of the human eyelid margins: an in vivo confocal and histological study. J Anat 2011;218:449-61

29. Norn M. Meibomian orifices and Marx's line. Studied by triple vital staining. Acta Ophthalmol (Copenh) 1985;63:698-700

30. Yamaguchi M, Kutsuna M, Uno T, et al. Marx line: fluorescein staining line on the inner lid as indicator of meibomian gland function. Am J Ophthalmol 2006;141:669-75

31. Bron AJ, Benjamin L, Snibson GR. Meibomian gland disease. Classification and grading of lid changes. Eye (Lond) 1991;5( Pt 4):395-411

32. Foulks $\mathrm{G}$, Bron AJ. A clinical description of meibomian gland dysfunction. Ocul Surf 2003:107-26

33. Keith CG. Seborrhoeic blepharo-kerato-conjunctivitis. Trans Ophthalmol Soc U K 1967;87:85-103

34. Meibomian Gland Dysfunction International Workshop Report. Invest Ophthalmol Vis Sci 2011, in press

35. Ehlers N. The precorneal film. Biomicroscopical, histological and chemical investigations. Acta Ophthalmol (Copenh) 1965;Suppl 81:79-136

36. Kessing SV. Mucous gland system of the conjunctiva. A quantitative normal anatomical study. Acta Ophthalmol (Copenh) 1968; Suppl 95:1-133

37. Lipham WJ, Tawfik HA, Dutton JJ. A histologic analysis and threedimensional reconstruction of the muscle of Riolan. Ophthal Plast Reconstr Surg 2002;18:93-8

38. Korb DR, Greiner JV, Herman JP, et al. Lid-wiper epitheliopathy and dry-eye symptoms in contact lens wearers. CLAO J 2002;28:211-6

39. Korb DR, Herman JP, Greiner JV, et al. Lid wiper epitheliopathy and dry eye symptoms. Eye Contact Lens 2005;31:2-8

40. McMonnies CW. Incomplete blinking: exposure keratopathy, lid wiper epitheliopathy, dry eye, refractive surgery, and dry contact lenses. Cont Lens Anterior Eye 2007;30:37-51

41. Shiraishi A, Yamanishi S, Yamamoto Y, et al. [Lid-wiper epitheliopathy in patients with dry eye symptoms]. Nippon Ganka Gakkai Zasshi 2009;113:596-600

42. Huang AJW, Tseng SCG, Kenyon KR. Paracellular permeability of corneal and conjunctival epithelia. Invest Ophthalmol Vis Sci 1989;30:684-9

43. Prausnitz MR, Noonan JS. Permeability of cornea, sclera, and conjuctiva: a literature analysis for drug delivery to the eye. J Pharm Sci 1998;87:1479-88

44. Tonjum AM. Permeability of horseradish peroxidase in rabbit corneal epithelium. Acta Ophthalmol (Copenh) 1974;52:650-8

45. Ban Y, Cooper LJ, Fullwood NJ, et al. Comparison of ultrastructure, tight junction-related protein expression and barrier function of human corneal epithelial cells cultivated on amniotic membrane with and without air-lifting. Exp Eye Res 2003;76:735-43

46. Yoshida Y, Ban Y, Kinoshita S. Tight junction transmembrane protein claudin subtype expression and distribution in human corneal and conjunctival epithelium. Invest Ophthalmol Vis Sci 2009;50:2103-8

47. Chung YB, Han K, Nishiura A, Lee VH. Ocular absorption of Pz-peptide and its effect on the ocular and systemic pharmacokinetics of topically applied drugs in the rabbit. Pharm Res 1998;15:1882-7

48. Bartlett JD, Jaanus SD: Clinical ocular pharmacology. Boston, Butterworth Heineman, 2008

49. Tsukita S, Furuse M, Itoh M. Multifunctional strands in tight junctions. Nat Rev Mol Cell Biol 2001;2:285-93

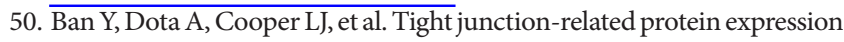
and distribution in human corneal epithelium. Exp Eye Res 2003;76:663-9

51. Furuse M, Hata M, Furuse K, et al. Claudin-based tight junctions are crucial for the mammalian epidermal barrier: a lesson from claudin-1deficient mice. J Cell Biol 2002;156:1099-111

52. Furuse $M$, Hirase $T$, Itoh $M$, et al. Occludin: a novel integral membrane protein localizing at tight junctions. J Cell Biol 1993;123:1777-88

53. Yu AS, McCarthy KM, Francis SA, et al. Knockdown of occludin expression leads to diverse phenotypic alterations in epithelial cells. Am J 
Physiol Cell Physiol 2005;288:C1231-41

54. Wang Z, Mandell KJ, Parkos CA, et al. The second loop of occludin is required for suppression of Raf1-induced tumor growth. Oncogene 2005;24:4412-20

55. Lajtha LG. Stem cell concepts. Nouv Rev Fr Hematol 1979;21:59-65

56. Barrandon Y. [The biology of epidermal stem cells]. Ann Dermatol Venereol 1998;125 Suppl 2:S5-6

57. Wei Z-G, Wu R-L, Lavker RM, Sun T-T. In vitro growth and differentiation of rabbit bulbar, fornix, and palpebral conjunctival epithelia. InvestOphthalmol Vis Sci 1993;34:1814-27

58. Wei ZG, Sun TT, Lavker RM. Rabbit conjunctival and corneal epithelial cells belong to two separate lineages. Invest Ophthalmol Vis Sci 1996;37:523-33

59. Pellegrini G, Golisano O, Paterna P, et al. Location and clonal analysis of stem cells and their differentiated progeny in the human ocular surface. J Cell Biol 1999;145:769-82

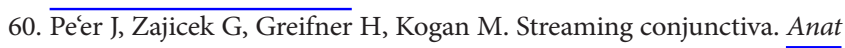
$\operatorname{Rec} 1996 ; 245: 36-40$

61. Wirtschafter JD, McLoon LK, Ketcham JM, et al. Palpebral conjunctival transient amplifying cells originate at the mucocutaneous junction and their progeny migrate toward the fornix. Trans Am Ophthalmol Soc 1997;95:417-29; discussion 29-32

62. Wirtschafter JD, Ketcham JM, Weinstock RJ, et al. Mucocutaneous junction as the major source of replacement palpebral conjunctival epithelial cells. Invest Ophthalmol Vis Sci 1999;40:3138-46

63. Tseng SCG. Concept and application of limbal stem cells. Eye 1989;3:141-57

64. Cotsarelis G, Cheng SZ, Dong G, et al. Existence of slow-cycling limbal epithelial basal cells that can be preferentially stimulated to proliferate: Implications on epithelial stem cells. Cell 1989;57:201

65. Lavker RM, Sun TT. Epidermal stem cells. J Invest Dermatol 1983;81:121-7

66. Cotsarelis G, Sun TT, Lavker RM. Label-retaining cells reside in the bulge area of pilosebaceous unit: implications for follicular stem cells, hair cycle, and skin carcinogenesis. Cell 1990;61:1329-37

67. Lavker RM, Miller S, Wilson C, et al. Hair follicle stem cells: their location, role in hair cycle, and involvement in skin tumor formation. J Invest Dermatol 1993;101:16S-26S

68. Leblond CP, Clermont Y, Nadler NJ. The pattern of stem cell renewal in three epithelia: (esophagus, intestine and testis). Proc Can Cancer Conf 1967;7:3-30

69. Rochat A, Kobayashi K, Barrandon Y. Location of stem cells of human hair follicles by clonal analysis. Cell 1994;76:1063-73

70. Dartt DA. Regulation of mucin and fluid secretion by conjunctival epithelial cells. Prog Retin Eye Res 2002;21:555-76

71. Nichols JJ, Mitchell GL, King-Smith PE. Thinning rate of the precorneal and prelens tear films. Invest Ophthalmol Vis Sci 2005;46:2353-61

72. Tsubota K. Tear dynamics and dry eye. Prog Retin Eye Res 1998;17:565-96

73. McDonald JE, Brubaker S. Meniscus-induced thinning of tear films. Am J Ophthalmol 1971;72:139-46

74. Miller KL, Polse KA, Radke CJ. Black-line formation and the „perched“ human tear film. Curr Eye Res 2002;25:155-62

75. Mainstone JC, Bruce AS, Golding TR. Tear meniscus measurement in the diagnosis of dry eye. Curr Eye Res 1996;15:653-61

76. Wang J, Simmons P, Aquavella J, et al. Dynamic distribution of artificial tears on the ocular surface. Arch Ophthalmol 2008;126:

77. Wang J, Palakuru JR, Aquavella JV. Correlations among upper and lower tear menisci, noninvasive tear break-up time, and the Schirmer test. Am J Ophthalmol 2008;145:795-800

78. Yokoi N, Bron A, Tiffany J, Kinoshita S. Reflective meniscometry a new field of dry eye assessment. Cornea 2000;19 (suppl 1):S37-43

79. Mishima S, Gasset A, Klyce S, Baum J. Determination of tear volume and tear flow. Invest Ophthalmol 1966;5:264-76

80. Yokoi N, Bron AJ, Tiffany JM, et al. Relationship between tear volume and tear meniscus curvature. Arch Ophthalmol 2004;122:1265-9

81. Liotet S, Van Bijsterveld OP, Kogbe O, Laroche L. A new hypothesis on tear film stability. Ophthalmologica 1987;195:119-24

82. Tiffany JM. Measurement of wettability of the corneal epithelium. 1. Particle attachment method. Acta Ophthalmol (Copenh) 1990;68:175-81

83. Tiffany JM. Measurement of wettability of the corneal epithelium. 2 . Contact angle method. Acta Ophthalmol (Copenh) 1990;68:182-7

84. Gipson IK, Spurr-Michaud SJ, Tisdale AS, et al. Stratified squamous epithelia produce mucin-like glycoproteins. Tissue Cell 1995;27:397-404

85. Argueso P, Gipson IK. Epithelial mucins of the ocular surface: structure, biosynthesis and function. ExpEye Res 2001;73:281-9

86. Argueso P, Balaram M, Spurr-Michaud S, et al. MUC 16 mucin is expressed by the human ocular surface epithelia and carries the H185 carbohydrate epitope. Invest Ophthalmol Vis Sci 2003;44:2487-95

87. Argueso P, Sumiyoshi M. Characterization of a carbohydrate epitope defined by the monoclonal antibody H185: sialic acid O-acetylation on epithelial cell-surface mucins. Glycobiology 2006;16:1219-28

88. Argueso P, Tisdale A, Spurr-Michaud S, et al. Mucin characteristics of human corneal-limbal epithelial cells that exclude the rose bengal anionic dye. Invest Ophthalmol Vis Sci 2006;47:113-9

89. Pfluger. Zu Ernahrung der cornea. Klin Monatsbl Augenheilkd 1882;20:69-81

90. Straub M. Fluoresceinlosung als ein diagnostisches Hilfsmittel fur Hornhauterkrankungen. Centralbl Augenheilkd 1888;12:75-7

91. Fromm, Groenouw. Ueber die diagnostische Verwendbarkeit der Fleorescinfarbung die Augenkrankungen. Arch Augenheilkd 1891;22:247-57

92. Schirmer O. Studien zur Physiologie und Pathologie der Tränenabsonderung und Tränenabfuhr. Graefes Arch Klin Exper Ophthalmol 1903;56:197-291

93. Norn MS. Vital staining of cornea and conjunctiva. Acta Ophthalmol (Copenh) 1962;40:389-401

94. Norn MS. Fluorescein vital staining of the cornea and conjunctiva. Studied by triple staining with fluorescein, rose bengal, and alcian blue. Acta Ophthalmol (Copenh) 1964;42:1038-45

95. Norn MS. [Vital staining of mucous membranes. Specific staining with fluorescein, rose bengal and alcian blue]. Ugeskr Laeger 1965;127:716-7

96. Norn MS. Vital staining of the canaliculus lacrimalis and the palpebral border (Marx' line). Acta Ophthalmol (Copenh) 1966;44:948-59

97. Norn MS. Vital staining of the cornea and conjunctiva; with a mixture of fluorescein and rose bengal. Am J Ophthalmol 1967;64:1078-80

98. Norn MS. Dead, degenerate, and living cells in conjunctival fluid and mucous thread. Acta Ophthalmol (Copenh) 1969;47:1102-15

99. Norn M. Rose bengal vital staining. Staining of cornea and conjunctiva by 10 percent rose bengal, compared with 1 percent. Acta Ophthalmologica (Copenh) 1970;48:546-59

100. Norn MS. Micropunctate fluorescein vital staining of the cornea. Acta Ophthalmol (Copenh) 1970;48:108-18

101. Norn MS. Vital staining of cornea and conjunctiva. Fluorescein-rose bengal mixture and tetrazolium-alcian blue mixture. Acta Ophthalmol (Copenh) 1972;Suppl 113:3-66

102. Eliason AJ, Maurice DM. Staining of the conjunctiva and conjunctival tear film. Br J Ophthalmol 1990;74:519-22

103. Feenstra RP, Tseng SC. What is actually stained by rose bengal? Arch Ophthalmol 1992;110:984-93

104. Feenstra RPG, Tseng SCG. Comparison of fluorescein and rose bengal staining. Ophthalmology 1992;99:605-17

105. Chodosh J, Dix RD, Howell RC, et al. Staining characteristics and antiviral activity of sulforhodamine B and lissamine green B. Invest Ophthalmol Vis Sci 1994;35:1046-58

106. Norn MS: External eye. Methods of examination. Copenhagen, Scriptor, 1983, ed 2

107. Tabery HM. Dual appearance of fluorescein staining in vivo of diseased 
human corneal epithelium. A non-contact photomicrographic study. Br J Ophthalmol 1992;76:43-4

108. Tabery HM. Toxic effect of rose bengal dye on the living human corneal epithelium. Acta Ophthalmol Scand 1998;76:142-5

109. Tabery HM. Corneal surface changes in keratoconjunctivitis sicca. Part I: The surface proper. A non-contact photomicrographic in vivo study in the human cornea. Eye (Lond) 2003;17:482-7

110. Watanabe H, Maeda N, Kiritoshi A, et al. Expression of a mucin-like glycoprotein produced by ocular surface epithelium in normal and keratinized cells. Am J Ophthalmol 1997;124:751-7

111. Watanabe H, Fabricant M, Tisdale AS, et al. Human corneal and conjunctival epithelia produce a mucin-like glycoprotein for the apical surface. Invest Ophthalmol Vis Sci 1995;36:337-44

112. Danjo Y, Watanabe H, Tisdale AS, et al. Alteration of mucin in human conjunctival epithelia in dry eye. Invest Ophthalmol Vis Sci 1998;39:2602-9

113. Pflugfelder SC, Tseng SC, Yoshino K, et al. Correlation of goblet cell density and mucosal epithelial membrane mucin expression with rose bengal staining in patients with ocular irritation. Ophthalmology 1997; 104:223-35

114. Yin BW, Lloyd KO. Molecular cloning of the CA125 ovarian cancer antigen: identification as a new mucin, MUC16. J Biol Chem 2001;276:27371-5

115. Guzman-Aranguez A, Mantelli F, Argueso P. Mucin-type O-glycans in tears of normal subjects and patients with non-Sjogren's dry eye. Invest Ophthalmol Vis Sci 2009;50:4581-7

116. Argueso P, Guzman-Aranguez A, Mantelli F, et al. Association of cell surface mucins with galectin-3 contributes to the ocular surface epithelial barrier. J Biol Chem 2009;284:23037-45

117. Blalock TD, Spurr-Michaud SJ, Tisdale AS, et al. Functions of MUC16 in corneal epithelial cells. Invest Ophthalmol Vis Sci 2007;48:4509-18

118. Ehrlich P. Ueber provocirte Fluorescenzerscheinungen am Auge. Dtsch Med Wochenschr 1882;8:35-6

119. Maurice D. The use of fluorescein in opthalmological research. The Jonas Friedenwald Memorial lecture. Invest Ophthalmol 1967;6:464-77

120. Bron AJ, Evans VE, Smith JA. Grading of corneal and conjunctival staining in the context of other dry eye tests. Cornea 2003;22:640-50

121. Koh S, Watanabe H, Hosohata J, et al. Diagnosing dry eye using a bluefree barrier filter. Am J Ophthalmol 2003;136:513-9

122. Wilson G, Ren $\mathrm{H}$, Laurent J. Corneal epithelial fluorescein staining. J Am Optom Assoc 1995;66:435-41

123. Tabery HM. Micropunctate fluorescein staining of the human corneal surface: microerosions or cystic spaces? A non-contact photomicrographic in vivo study. Acta Ophthalmol Scand 1997;75:134-6

124. Korb DR, Blackie CA. Marxs line of the upper lid is visible in upgaze without lid eversion. Eye Contact Lens 2010;36:149-51

125. Korb DR, Blackie CA. Meibomian gland diagnostic expressibility: correlation with dry eye symptoms and gland location. Cornea 2008;27:1142-7
126. Doane MG. Interactions of eyelids and tears in corneal wetting and dynamics of the normal human eyeblink. Am J Ophthalmol 1980;89:507-16

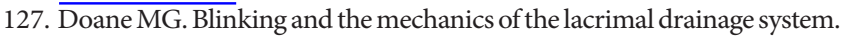
Ophthalmology 1981;88:844-51

128. Nakamori K, Odawara M, Nakajima T, et al. Blinking is controlled primarily by ocular surface conditions. Am J Ophthalmol 1997;124:24-30

129. Fatt I. Observations of tear film break up on model eyes. CLAO J 1991;17:267-81

130. Batchelor G: Introduction to fluid dynamics: Cambridge University Press, 1973

131. Lucas M. Estimation of sodium chloride diffusion coefficient in gastric nucin. Dig Dis Sci 1984;29:336-45

132. Spurr-Michaud S, Argueso P, Gipson I. Assay of mucins in human tear fluid. Exp Eye Res 2007;84:939-50

133. Riquelme R, Lira I, Pierez-Liopez CS, et al. Interferometric measurement of a diffusion coefficient: comparison of two methods and uncertainty analysis. J Phys Appl Phys 2007;40:2769-76

134. King-Smith PE, Nichols JJ, Nichols KK, et al. Contributions of evaporation and other mechanisms to tear film thinning and break-up. Optom Vis Sci 2008;85:623-30

135. King-Smith PE, Fink BA, Nichols JJ, et al. The contribution of lipid layer movement to tear film thinning and breakup. Invest Ophthalmol Vis Sci 2009;50:2747-56

136. Gilbard JP, Farris RL, Santa Maria J. Osmolarity of tear microvolumes in keratoconjunctivitis sicca. Arch Ophthalmol 1978;96:677-81

137. Gilbard JP, Carter JB, Sang DN, et al. Morphologic effect of hyperosmolarity on rabbit corneal epithelium. Ophthalmology 1984;91:1205-12

138. Gilbard JP, Rossi SR. Changes in tear ion concentration in dry-eye disorders. Adv Exp Med Biol 1994;350:529-33

139. Gilbard JP, Farris RL. Tear osmolarity and ocular surface disease in keratoconjunctivitis sicca. Arch Ophthalmol 1979;97:1642-6

140. Lawrenson JG, Birhah R, Murphy PJ. Tear-film lipid layer morphology and corneal sensation in the development of blinking in neonates and infants. J Anat 2005;206:265-70

141. Crowther R, Marriott C. Counter-ion binding to mucus glycoproteins. J Pharm Pharmacol 1984;36:21-6

142. Varma B, Demers A, Jamieson A, et al. Light scattering studies of the effect of $\mathrm{Ca} 2+$ on the structure of porcine submaxillary mucin. Biopolymers 1990;29:441-8

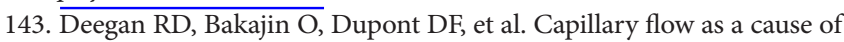
ring stains from dried liquid drops. Nature 1997;389:827-9

144. Barrat J, Hansen J: Basic concepts for simple and complex liquids: Cambridge, Cambridge University Press, 2003

145. Taylor G: A movie of kinematic reversibility in Stokes flow, in Homsy GM (ed). Multi-media fluid mechanics. Cambridge, Cambridge University Press, 1999

146. Arratia P, Voth G, Gollub J. Stretching and mixing of non-Newtonian fluids in time-periodic flows. PhysFluids 17:2005:053102 